\title{
TRIANGULATION OF THE MAP OF A $G$-MANIFOLD TO ITS ORBIT SPACE
}

\author{
MITSUTAKA MURAYAMA AND MASAHIRO SHIOTA
}

\begin{abstract}
Let $G$ be a Lie group, and let $M$ be a smooth proper $G$-manifold. Let $M / G$ denote the orbit space, and let $\pi: M \rightarrow M / G$ be the natural map. It is known that $M / G$ is homeomorphic to a polyhedron. In the present paper we show that there exist a piecewise linear (PL) manifold $P$, a polyhedron $L$, and homeomorphisms $\tau: P \rightarrow M$ and $\sigma: M / G \rightarrow L$ such that $\sigma \circ \pi \circ \tau$ is PL. This is an application of the theory of subanalytic sets and subanalytic maps of Shiota. If $M$ and the $G$-action are, moreover, subanalytic, then we can choose $\tau$ and $\sigma$ subanalytic and $P$ and $L$ unique up to PL homeomorphisms.
\end{abstract}

\section{$\S 1$. Introduction}

Let $G$ be a Lie group, which we regard as of analytic class. A $C^{k} G$ manifold $M(k=1, \ldots, \infty, \omega)$ is a $C^{k}$ manifold with an action of $G$ on $M$ such that the map $G \times M \ni(g, x) \rightarrow g x \in M$ is of class $C^{k}$. A manifold means a paracompact manifold without boundary, although our arguments are also valid in the case of paracompact manifolds with boundary. A $C^{k}$ $G$-manifold $M$ is called proper if the map $G \times M \ni(g, x) \rightarrow(x, g x) \in M^{2}$ is proper. Let $M / G$ denote the orbit space $\{G x \mid x \in M\}$ equipped with the quotient topology, and let $\pi: M \rightarrow M / G$ be the natural map. A triangulation of a topological space $X$ is a pair consisting of a polyhedron $P$ and a homeomorphism $\tau: P \rightarrow X$. We also call the homeomorphism $\tau: P \rightarrow X$ a triangulation of $X$. A triangulation of a $C^{0}$ map between topological spaces $\phi: X \rightarrow Y$ is a pair consisting of triangulations $\tau_{X}: P_{X} \rightarrow X$ of $X$ and $\tau_{Y}: P_{Y} \rightarrow Y$ of $Y$ such that the map $\tau_{Y}^{-1} \circ \phi \circ \tau_{X}: P_{X} \rightarrow P_{Y}$ is piecewise linear $(\mathrm{PL})$ (in the sense that there are simplicial decompositions $K_{X}$ of $P_{X}$ and $K_{Y}$ of $P_{Y}$ such that $\tau_{Y}^{-1} \circ \phi \circ \tau_{X}: K_{X} \rightarrow K_{Y}$ is simplicial). A $C^{k}$ triangulation of a $C^{k}$ manifold possibly with corners $N$ is a triangulation $\tau: P \rightarrow N$ of $N$ such that for some simplicial decomposition $K$ of $P$,

Received March 30, 2010. Revised October 3, 2012. Accepted December 12, 2012.

First published online September 5, 2013.

2010 Mathematics Subject Classification. Primary 57S15; Secondary 57S20, 58K20. 
the restriction of $\tau$ to each simplex in $K$ is a $C^{k}$ diffeomorphism onto its image. There always exists a $C^{k}$ triangulation of $N$, and the polyhedron $P$ is a PL manifold possibly with boundary and unique up to PL homeomorphisms, which is known as the Cairns-Whitehead theorem (this theorem is the object of the main theorem in [11]). The uniqueness is important for us. We naturally define a $C^{k}$ triangulation of a $C^{k}$ map between $C^{k}$ manifolds possibly with corners and can extend, by the arguments in [15], the CairnsWhitehead theorem to a theorem which states that, given a $C^{k}$ submersion $\phi: X \rightarrow Y$ between $C^{k}$ manifolds, possibly with corners such that $\phi$ carries each face of $X$ to some face of $Y$ and a $C^{k}$ triangulation $\tau_{Y}: P_{Y} \rightarrow Y$, we can lift $\tau_{Y}$ to a $C^{k}$ triangulation $\tau_{X}: P_{X} \rightarrow X$ in a weak sense that there is a simplicial decomposition $K_{X}$ of $P_{X}$ such that the restriction of $\tau_{Y}^{-1} \circ \phi \circ \tau_{X}$ to each simplex in $K_{X}$ is linear. We call $\left(\tau_{X}: P_{X} \rightarrow X, \tau_{Y}: P_{Y} \rightarrow Y\right)$ a $C^{k}$ triangulation of $\phi: X \rightarrow Y$ in the weak sense, and we call $\tau_{X}: P_{X} \rightarrow X$ a lift in the weak sense of $\tau_{X}: P_{Y} \rightarrow Y$. If $\phi$ is, moreover, a $C^{k}$ fiber bundle, that is, if each point of $Y$ has a neighborhood $U$ in $Y$ such that the map $\left.\phi\right|_{\phi^{-1}(U)}: \phi^{-1}(U) \rightarrow U$ is $C^{k}$ trivial and the fibers $\phi^{-1}(y)$ and $\phi^{-1}\left(y^{\prime}\right)$ are $C^{k}$ diffeomorphic for any points $y, y^{\prime} \in Y$, we can choose the triangulation $\tau_{X}: P_{X} \rightarrow X$ so that $\tau_{Y}^{-1} \circ \phi \circ \tau_{X}$ is PL (see [15, Lemma 1]). Then we call $\tau_{X}: P_{X} \rightarrow X$ a lift of $\tau_{X}: P_{Y} \rightarrow Y$. We call this generalization the Cairns-Whitehead theorem.

Triangulations (not just triangulability) of orbit spaces are studied by many people (e.g., Matumoto and Shiota [8], [9], Verona [17], and Yang [19]). In the present paper, we show a triangulation of the map $\pi: M \rightarrow M / G$.

THEOREM 1.1. Let $G$ be a Lie group, and let $M$ be a proper $C^{k} G$ manifold, $k=1, \ldots, \infty, \omega$. Then there exists a triangulation $(\tau: P \rightarrow M, \sigma:$ $L \rightarrow M / G)$ of the map $\pi: M \rightarrow M / G$ such that $P$ is the $P L$ manifold of a $C^{k}$ triangulation of $M$. Moreover, if $M$ is a subanalytic $C^{k} G$-manifold, then we can choose the triangulations $\tau: P \rightarrow M$ and $\sigma: L \rightarrow M / G$ subanalytic, and $P$ and $L$ are unique up to $P L$ homeomorphisms.

It is not difficult to prove Theorem 1.1 locally. To be precise, each point of $M / G$ has a neighborhood $U$ such that the restriction $\left.\pi\right|_{\pi^{-1}(U)}: \pi^{-1}(U) \rightarrow U$ is triangulable. The problem is how to paste local triangulations, and we need to find local triangulations so that we can paste them. Let $U_{1}$ and $U_{2}$ be open subsets of $M / G$ such that there are triangulations $\left(\tau_{i}: P_{i} \rightarrow\right.$ $\left.\pi^{-1}\left(U_{i}\right), \sigma_{i}: L_{i} \rightarrow U_{i}\right)$ of the maps $\left.\pi\right|_{\pi^{-1}\left(U_{i}\right)}: \pi^{-1}\left(U_{i}\right) \rightarrow U_{i}, i=1,2$. Set $U=U_{1} \cup U_{2}$. Then we need to find a triangulation of the map $\left.\pi\right|_{\pi^{-1}(U)}$ : 
$\pi^{-1}(U) \rightarrow U$. For that we will choose the two triangulations so that the polyhedra $\tau_{1}^{-1}\left(\pi^{-1}(x)\right)$ and $\tau_{2}^{-1}\left(\pi^{-1}(x)\right)$ are PL homeomorphic for each $x \in U_{1} \cap U_{2}$; that is, the two triangulations of each $\pi^{-1}(x)$ are unique up to $\mathrm{PL}$ homeomorphisms. This is a natural minimum requirement for pasting of two triangulations. Let us consider the problem more generally.

Problem 0. Let $\phi: X \rightarrow Y$ be a $C^{0}$ map between topological spaces in Euclidean spaces. Assume that each point of $Y$ has a neighborhood $U$ such that the restriction $\left.\phi\right|_{\phi^{-1}(U)}: \phi^{-1}(U) \rightarrow U$ is triangulable. Is $\phi$ triangulable?

Thom [16] conjectured that the problem is correct if $\phi$ is proper and satisfies a condition, and the second author solved the conjecture (see [15, Theorem]). Roughly speaking, the condition says that when a point $y$ moves in $Y$, the fiber $\phi^{-1}(y)$ does not explode, and if $X$ and $Y$ are complex nonsingular algebraic varieties and $\phi$ is a morphism, then the condition is related to the condition that $\phi$ is flat. Thom called $\phi$ satisfying the condition une application sans éclatement, and we call it a Thom map. We will prove that the map $\pi: M \rightarrow M / G$ is a Thom map. However, $\pi$ is not necessarily proper, and a nonproper Thom map is not necessarily triangulable. Hence, for the proof of Theorem 1.1, it is not sufficient that we regard $\pi$ as a general Thom map. We use the condition of subanalyticity on $\pi$. Indeed, if $\pi$ is a subanalytic Thom map, then we can prove Theorem 1.1. Thus, we will first give subanalytic structures to the sets $M, M / G$ and the map $\pi: M \rightarrow M / G$, and we will then prove Theorem 1.1.

Before giving subanalytic structures, we reduce Theorem 1.1 to the case where the manifolds $G$ and $M$ satisfy the second countability axiom; that is, they can be embedded in some Euclidean spaces because a subanalytic set is a subset of a Euclidean space. Choose a family $\left\{M_{\alpha}\right\}_{\alpha \in A}$ of connected components of $M$ so that $M$ is the disjoint union of $G M_{\alpha}, \alpha \in A$, and set $G_{\alpha}=\left\{g \in G \mid g M_{\alpha}=M_{\alpha}\right\}$. Then each $G_{\alpha}$ is a second-countable Lie group, and each $M_{\alpha}$ is a second-countable $C^{k} G_{\alpha}$-manifold (see [5, Remark 2]). Define the natural map $\pi_{\alpha}: M_{\alpha} \rightarrow M_{\alpha} / G_{\alpha}$. Then the family $\left\{M_{\alpha} / G_{\alpha}\right\}_{\alpha \in A}$ is the connected components of $M / G$. Hence, we can assume that $A$ is a singleton, that is, that $M / G=M_{\alpha} / G_{\alpha}$. Moreover, we reduce Theorem 1.1 to the case $M=M_{\alpha}$ as follows.

Suppose that Theorem 1.1 is proved for $M_{\alpha}$ and $G_{\alpha}$, and let $\left(\tau_{\alpha}: P_{\alpha} \rightarrow\right.$ $\left.M_{\alpha}, \sigma_{\alpha}: L_{\alpha} \rightarrow M_{\alpha} / G_{\alpha}\right)$ be a resulting triangulation of the map $\pi_{\alpha}: M_{\alpha} \rightarrow$ $M_{\alpha} / G_{\alpha}$. We need to extend the triangulation $\tau_{\alpha}: P_{\alpha} \rightarrow M_{\alpha}$ to a triangulation $\tau: P \rightarrow M$. For this it suffices to define a triangulation $\tau_{0}: P_{0} \rightarrow M_{0}$ 
of a connected component $M_{0}$ of $M$ so that the map $\sigma_{\alpha}^{-1} \circ \pi \circ \tau_{0}$ is PL. Choose an element $g_{0}$ of $G$ so that $g_{0} M_{\alpha}=M_{0}$, set $P_{0}=P_{\alpha}$, and define $\tau_{0}$ by $\tau_{0}(x)=g_{0} \tau_{\alpha}(x)$ for $x \in P_{0}$. Then the map $\tau_{0}: P_{0} \rightarrow M_{0}$ is a triangulation of $M_{0}$, and the map $\sigma_{\alpha}^{-1} \circ \pi \circ \tau_{0}$ is PL, because

$$
\sigma_{\alpha}^{-1} \circ \pi \circ \tau_{0}(x)=\sigma_{\alpha}^{-1} \circ \pi\left(g_{0} \tau_{\alpha}(x)\right)=\sigma_{\alpha}^{-1} \circ \pi_{\alpha} \circ \tau_{\alpha}(x) \text { for } x \in P_{0} .
$$

The additional condition in Theorem 1.1 that $P$ is the PL manifold of a $C^{k}$ triangulation of $M$ is obvious by the above arguments. Thus, we assume that the second countability axiom is satisfied.

We can give an analytic $G$-manifold structure to $M$ and embed $M / G$ into some Euclidean space $\mathbf{R}^{n}$ so that the map $\pi: M \rightarrow \mathbf{R}^{n}$ is analytic, as we will see. However, we cannot give an analytic manifold structure or an analytic set structure to $M / G$. Hence, we cannot use theories of analytic sets and analytic maps. We proceed in the category of subanalytic sets and maps instead. A subanalytic set is a subset of a Euclidean space $\mathbf{R}^{n}$ of the form $\bigcup_{i}\left(\operatorname{Im} f_{i 1}-\operatorname{Im} f_{i 2}\right)$, where $f_{i j}$ are a finite number of proper real analytic maps from real analytic manifolds into $\mathbf{R}^{n}$, and a subanalytic map is a continuous map between subanalytic sets with subanalytic graph.

It is known that a subanalytic set closed in the ambient Euclidean space is subanalytically homeomorphic to a polyhedron (van der Waerden-GieseckeŁojasiewicz; see [7, Theorem 1]). However, a subanalytic map between subanalytic sets closed in the ambient Euclidean spaces is not necessarily triangulable. For example, a blowing-up $\mathbf{R}^{2} \ni(x, y) \rightarrow(x, x y) \in \mathbf{R}^{2}$ is not triangulable, which is une application avec éclatement. In [14, Chapter IV], the second author considered when subanalytic maps are triangulable. An open problem is problem 0 for a subanalytic map. Problem 0 in the subanalytic case looks true because a subanalytic triangulation of a subanalytic set is unique up to PL homeomorphisms (the subanalytic Hauptvermutung [14, Corollary III.1.4]), and hence, a subanalytic triangulation of the set $\phi^{-1}(y)$ is unique for $\phi$ and each $y \in Y$ in problem 0 . On the other hand, a triangulation of a triangulable topological set is not unique (failure of the Hauptvermutung found by Milnor in [10, Theorem 1.2]), and hence, problem 0 may be false. By these facts, we give subanalytic structures to the sets $M, M / G$ and the map $\pi: M \rightarrow M / G$ to prove Theorem 1.1. We use a theory of subanalytic sets and subanalytic maps, which is close to a theory of o-minimal structures in model theory (see [14]). We also use the CairnsWhitehead theorem since a $C^{k}$ triangulation of a $C^{k}$ manifold possibly with corners is unique. 
We will prove the following statements in order. First, the manifold $M$ in Theorem 1.1 is equivariantly $C^{k}$ diffeomorphic to a subanalytic analytic $G$-manifold $M_{1}$ (see Lemma 3.1). Next, $M_{1} / G$ is embedded in a Euclidean space so that its image is subanalytic and closed and the natural map $\pi_{1}$ : $M_{1} \rightarrow M_{1} / G$ is subanalytic with respect to the subanalytic structure on the image (the proof of Lemma 3.2). Lastly, the map $\pi_{1}: M_{1} \rightarrow M_{1} / G$ admits a subanalytic triangulation $\left(\tau_{1}: P_{1} \rightarrow M_{1}, \sigma_{1}: L_{1} \rightarrow M_{1} / G\right)$. Assume that we have proved these. Then $P_{1}$ and $L_{1}$ are unique up to PL homeomorphisms by the subanalytic Hauptvermutung, the latter half of Theorem 1.1 is proved, and there is a PL homeomorphism $\tau_{2}$ from $P_{1}$ to some polyhedron $P_{2}$ such that the map $\tau_{1} \circ \tau_{2}^{-1}: P_{2} \rightarrow M_{1}$ is a subanalytic $C^{k}$ triangulation of $M_{1}$ by the subanalytic Hauptvermutung and the Cairns-Whitehead theorem ((12) in Section 2). Thus, the former half of Theorem 1.1 is proved.

We do not know whether the triangulation map $\sigma^{-1} \circ \pi \circ \tau: P \rightarrow L$ is unique in the latter half of Theorem 1.1, that is, whether for another subanalytic triangulation $\left(\tau^{\prime}: P^{\prime} \rightarrow M, \sigma^{\prime}: L^{\prime} \rightarrow M / G\right)$ of $\pi$, there exist PL homeomorphisms $\phi: P \rightarrow P^{\prime}$ and $\psi: L \rightarrow L^{\prime}$ such that $\psi \circ \sigma^{-1} \circ \pi \circ \tau=$ $\sigma^{\prime-1} \circ \pi \circ \tau^{\prime} \circ \phi$. Another open problem is whether we can choose a triangulation $(\tau: P \rightarrow M, \sigma: L \rightarrow M / G)$ of $\pi$ so that for each element $g$ of $G$, the action $P \ni x \rightarrow \tau^{-1}(g \tau(x)) \in P$ is PL.

Theorem 1.1 remains true in the case where $M$ is a proper $C^{k} G$-manifold with boundary as follows. Naturally, we give a proper $C^{k} G$-manifold structure to the double $D M$ of $M$, and we consider the pair of $D M$ and $\partial M$. Then it suffices to generalize Theorem 1.1 to the following form. In Theorem 1.1, let $M^{\prime}$ be a proper $C^{k} G$-submanifold of $M$, and assume that $M^{\prime}$ is closed in $M$. Then we can choose the triangulation $(\tau: P \rightarrow M, \sigma: L \rightarrow M / G)$ so that $\tau^{-1}\left(M^{\prime}\right)$ is a subpolyhedron of $P$. This is clear by the accompanying proof.

\section{$\S 2$. Subanalytic sets and Whitney stratifications}

Note that a subanalytic set is always embedded in some Euclidean space, and hence we regard two subanalytic sets as distinct if they are subanalytically homeomorphic but different from each other as subsets of Euclidean spaces. Examples of a subanalytic set and a subanalytic map are a polyhedron included in a Euclidean space as a closed subset and a PL map between such polyhedra, respectively. Note that an analytic submanifold of $\mathbf{R}^{n}$ and an analytic map between subanalytic analytic submanifolds of $\mathbf{R}^{n}$ are not 
necessarily subanalytic; for example, the set $\left\{(x, y) \in \mathbf{R}^{2} \mid x \neq 0, y=\sin 1 / x\right\}$ is an analytic submanifold of $\mathbf{R}^{2}$ but not subanalytic because a subanalytic set does not infinitely oscillate around a point. A sufficient condition for them to be subanalytic is that the submanifolds are included in Euclidean spaces as closed subsets. We call this sufficient condition the closed condition. A subset $X$ of $\mathbf{R}^{n}$ is subanalytic if and only if $X$ is subanalytic locally at each point of $\mathbf{R}^{n}$, but $X$ is analytic if and only if $X$ is analytic locally at each point of $X$. It is known that a $C^{k}$ manifold admits an analytic manifold structure (see [18, Theorem 1]), and that an analytic manifold $N$ is analytically embedded in a Euclidean space (see [3, Theorem 2]). Moreover, we can choose the embedding proper because any $C^{0}$ function on $N$ is approximated by an analytic function in the uniform topology (see [18, Theorem 2]), and hence, there exists a proper analytic function on $N$. Therefore, we can embed $N$ into some Euclidean space, so that the closed condition is satisfied, and give a subanalytic structure to $N$. We can choose the Euclidean space of dimension $=2 \operatorname{dim} N+1$ (see [18, Theorem 1]). We always assume that $G$ satisfies the closed condition, though some of the analytic submanifolds below do not satisfy it. We also assume that polyhedra are included in Euclidean spaces satisfying the closed condition and hence that they are subanalytic. Triangulations of a subanalytic set and a subanalytic map are called subanalytic if the homeomorphisms of the triangulations are subanalytic. We define a subanalytic $C^{k} G$-manifold $M$ by requiring that $M$ and the map $G \times M \rightarrow M$ be subanalytic. We need notions of a subanalytic $C^{k}$ manifold and a subanalytic $C^{k}$ map for finite $k$ because we use a partition of unity for construction of controlled tube systems, which we define later. An analytic partition of unity does not exist. This is another reason why we work in the subanalytic category but not in the analytic category.

It is an easy task to see that the map $\pi: M \rightarrow M / G$ is a Thom map. Hence, in the case of compact $G$, the map $\pi$ is proper and Theorem 1.1 follows. However, if $G$ is not compact, then $\pi$ is nonproper, and a nonproper Thom map is not necessarily triangulable, as we will show in an example. We modify Thom's conjecture as in Theorem 2.1 below. For that we need to proceed in the subanalytic category, as we have already explained. We prepare some terminology and facts (see [2, Chapter II] for Whitney stratifications and tube systems, [4] and [14] for elementary properties of subanalytic sets, and [14] for the subanalytic Whitney stratifications and tube systems). 
Thom introduced a theory of Whitney stratifications and tube systems to investigate polynomial and analytic maps [16]. However, he abstracted analyticity from the theory, and the theory works on some generic $C^{k}$ maps, $k>0$. On the other hand, we need subanalyticity, and we use a combination of a theory of subanalytic sets and maps and a theory of Whitney stratifications and tube systems.

For a subanalytic set $X \subset \mathbf{R}^{n}$, let $\operatorname{Reg} X$ denote the subset consisting of points where the germ of $X$ is analytic, smooth, and of maximal dimension. For a subanalytic map $\phi$ from a subanalytic analytic manifold $X_{1}$ to a subanalytic set $Y$, let $\operatorname{Reg} \phi$ denote the points of $X_{1}$ where the germ of $\phi$ is analytic and smooth and locally has the maximal Jacobian rank. Then $\operatorname{Reg} \phi$ is not necessarily a manifold, and its connected components are analytic manifolds of various dimension. We call such a set an analytic manifold of various local dimension. A subanalytic $C^{k}$ foliation, $k>0$, of a subanalytic $C^{k}$ manifold $X_{1}$ in $\mathbf{R}^{n}$ is a $C^{k}$ foliation $\mathfrak{F}$ which is subanalytically trivial locally at each point of $\mathbf{R}^{n}$ (not $X_{1}$ ). To be precise, each point of $\mathbf{R}^{n}$ has a subanalytic neighborhood $U$ in $\mathbf{R}^{n}$, and there is a subanalytic $C^{k}$ diffeomorphism $\phi: X_{1} \cap U \rightarrow(0,1)^{n_{1}}$ (not $\mathbf{R}^{n_{1}}$ ) such that $\{\phi(L \cap U) \mid L \in \mathfrak{F}\}=\left\{\{a\} \times(0,1)^{n_{2}} \mid a \in(0,1)^{n_{1}-n_{2}}\right\}$ for some $n_{1}$ and $n_{2}$. We say that two subanalytic analytic manifolds $X_{1}$ and $X_{2}$ in $\mathbf{R}^{n}$ satisfy the Whitney condition at a point $b$ of the set $X_{2} \cap \overline{X_{1}}$ if the following statement is true.

Let $\left\{a_{k}\right\}_{k=1,2, \ldots}$ and $\left\{b_{k}\right\}_{k=1,2, \ldots}$ be sequences of points in $X_{1}$ and $X_{2}$, respectively, both converging to $b$ such that the sequence of the tangent spaces $\left\{T_{a_{k}} X_{1}\right\}_{k=1,2, \ldots}$ converges to a subspace $T \subset \mathbf{R}^{n}$ in $G_{n, m}$ - the Grassmannian of $m$-dimensional subspaces of $\mathbf{R}^{n}$-where $m=\operatorname{dim} X_{1}$, and the sequence of the lines $\left\{\overrightarrow{a_{k} b_{k}}\right\}_{k=1,2, \ldots}$ converges to a line $L \subset \mathbf{R}^{n}$ in $G_{n, 1}$. Then $L \subset T$.

We note elementary properties of subanalytic sets which we use (see [4] and [14]). Let $X$ and $Y$ be subanalytic subsets of $\mathbf{R}^{n}$, let $X_{1}$ and $X_{2}$ be subanalytic analytic submanifolds of $\mathbf{R}^{n}$ such that $X_{2} \subset \overline{X_{1}}-X_{1}$, let $f$ : $\mathbf{R}^{n} \rightarrow \mathbf{R}^{n}$ be a subanalytic map, let $\phi: X_{1} \rightarrow Y$ be a subanalytic map, and let $k>0$. Then

(1) $X \cap Y, X \cup Y, X \times Y, X-Y$ and $\bar{X}$ are subanalytic;

(2) $\bar{X}-X$ is of dimension smaller than $X$ for nonempty $X$;

(3) $\operatorname{Reg} X$ is subanalytic;

(4) $\operatorname{dim}(X-\operatorname{Reg} X)<\operatorname{dim} X$ for nonempty $X$; 
(5) the family of connected components of $X$ is finite locally at each point of $\mathbf{R}^{n}$;

(6) each connected component is subanalytic;

(7) the subset of $X_{2}$ where $X_{1}$ and $X_{2}$ satisfy the Whitney condition is subanalytic;

(8) its complement in $X_{2}$ is of dimension smaller than $\operatorname{dim} X_{2}$ for nonempty $X_{2}$;

(9) $\operatorname{Reg} \phi$ is subanalytic;

(10) $\operatorname{dim}\left(X_{1}-\operatorname{Reg} \phi\right)<\operatorname{dim} X_{1}$ for nonempty $X_{1}$;

(11) the image $f(X)$ is subanalytic when $X$ is bounded or the restriction $\left.f\right|_{X}$ is a homeomorphism onto its image and the image is closed in $\mathbf{R}^{n}$

(12) a subanalytic $C^{k}$ manifold possibly with corners satisfying the closed condition is subanalytically $C^{k}$ triangulable, and for a subanalytic $C^{k}$ fiber bundle $X \rightarrow Y$, any subanalytic $C^{k}$ triangulation $\tau_{Y}: P_{Y} \rightarrow Y$ is lifted to a subanalytic $C^{k}$ triangulation $\tau_{X}: P_{X} \rightarrow X$ if $X$ and $Y$ are subanalytic $C^{k}$ manifolds with possibly corners satisfying the closed condition (we call this also the Cairns-Whitehead theorem);

(13) the family $\left\{\phi^{-1}(y) \mid y \in Y\right\}$ is a subanalytic $C^{k}$ foliation of $X_{1}$ if $Y$ is a bounded subanalytic $C^{k}$ manifold possibly with corners and $\phi$ is a $C^{k}$ submersion onto $Y$; and

(14) the normal bundle of a subanalytic $C^{k}$ foliation $\mathfrak{F}$ of $X_{1}(k>1)$ is subanalytic in the sense that there exists a subanalytic $C^{k-1}$ submanifold $U$ of $X_{1} \times \mathbf{R}^{n}$ of the form $\bigcup_{x \in X_{1}}\{x\} \times U_{x}$ such that for each $L \in \mathfrak{F}$, the map $\bigcup_{x \in L}\{x\} \times U_{x} \supset\{x\} \times U_{x} \ni\left(x, x^{\prime}\right) \rightarrow x \in L$ is the normal bundle of $L$ in $\mathbf{R}^{n}$.

First, we consider stratifications of subanalytic sets. A subanalytic analytic stratification $\left\{X_{i}\right\}_{i=1,2, \ldots}$ of a subanalytic set $X$ is a partition of $X$ into a finite number of subanalytic analytic manifolds $X_{i}$. We note by (1), (5), and (6) that for a bounded open subanalytic subset $U$ of the ambient Euclidean space of $X$, the family of the connected components of all $X_{i} \cap U$ is a subanalytic analytic stratification of $X \cap U$. By (1), (3), and (4), the family $\{\operatorname{Reg} X, \operatorname{Reg}(X-\operatorname{Reg} X), \ldots\}$ is a subanalytic analytic stratification of $X$. Set $X_{1}=\operatorname{Reg} X, X_{2}=\operatorname{Reg}(X-\operatorname{Reg} X) \ldots$ This stratification is canonical in the sense that for another subanalytic analytic stratification $\left\{X_{i}^{\prime}\right\}_{i}$ of $X$ distinct from $\left\{X_{i}\right\}_{i}$ and satisfying the condition $\operatorname{dim} X_{i_{1}}^{\prime} \geq \operatorname{dim} X_{i_{2}}^{\prime}\left(i_{1}<\right.$ $i_{2}$ ), either $X_{1} \supsetneq X_{1}^{\prime}$ or $X_{1}=X_{1}^{\prime}, \ldots, X_{i}=X_{i}^{\prime}$ and $X_{i+1} \supsetneq X_{i+1}^{\prime}$ for some $i$. 
A Whitney subanalytic analytic stratification $\left\{X_{i}\right\}_{i}$ is the case where each pair of $X_{i}$ and $X_{i^{\prime}}$ satisfies the Whitney condition at each point of $X_{i^{\prime}} \cap$ $\overline{X_{i}}$. By (1), (2), (7), and (8) there exists a Whitney subanalytic analytic stratification $\left\{X_{i}\right\}_{i}$ of $X$. We explicitly construct a Whitney subanalytic analytic stratification as follows. Let $X_{1}$ be $\operatorname{Reg} X$; let $X_{2}$ be the union of the sets $A_{1}$ and $A_{2}$ if $A_{1}$ and $A_{2}$ are of the same dimension, where $A_{1}$ is $\operatorname{Reg}(X-$ $\operatorname{Reg} X)-\overline{\operatorname{Reg} X}$ and $A_{2}$ is the subset of $\operatorname{Reg}(\operatorname{Reg}(X-\operatorname{Reg} X) \cap \overline{\operatorname{Reg} X})$, where $\operatorname{Reg} X$ and $\operatorname{Reg}(X-\operatorname{Reg} X)$ satisfy the Whitney condition, $X_{2}, A_{1}$, or $A_{2}$ of larger dimension unless $A_{1}$ and $A_{2}$ are of the same dimension; let $X_{3} \ldots$, and so on. Then this stratification is canonical in the same sense as the canonical subanalytic analytic stratification.

A typical example of a subanalytic set whose canonical subanalytic analytic stratification does not satisfy the Whitney condition is the Whitney umbrella $X=\left\{(x, y, z) \mid x^{2}=z y^{2}\right\}$ (see Figure 1). The canonical subanalytic analytic stratification of $X$ consists of $X_{2}$ the $z$-axis and $X_{1}$ its complement. The tangent space of $X_{1}$ at $\left(0, y_{0}, 0\right)$ is the $(x, y)$-plane for $y_{0} \neq 0$. On the other hand, there is a point $\left(0,0, y_{0}\right)$ in $X_{2}$, and the line $\overrightarrow{\left(0, y_{0}, 0\right)\left(0,0, y_{0}\right)}$ in $G_{n, 1}$ is $\mathbf{R}(0,1,-1)$. Hence, the Whitney condition is not satisfied for $\left\{X_{1}, X_{2}\right\}$. A typical example of a Whitney subanalytic analytic stratification is $\left\{\int \sigma \mid \sigma \in K\right\}$ for a finite simplicial complex $K$ in a Euclidean space. This example has the property that if $\int \sigma_{1} \cap\left(\overline{\int \sigma}-\int \sigma\right) \neq \emptyset$, then $\int \sigma_{1} \subset\left(\overline{\int \sigma}-\int \sigma\right)$. We call this property the frontier condition. In general, a

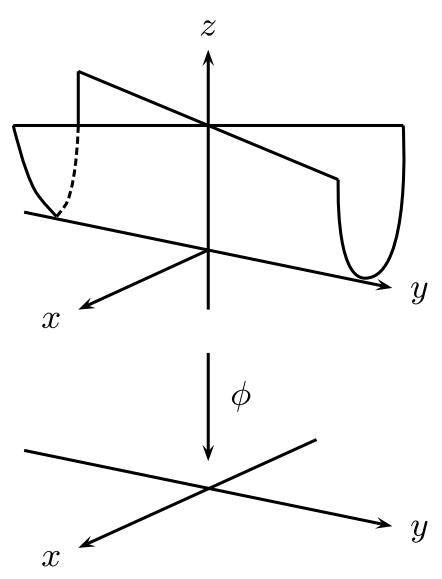

Figure 1 


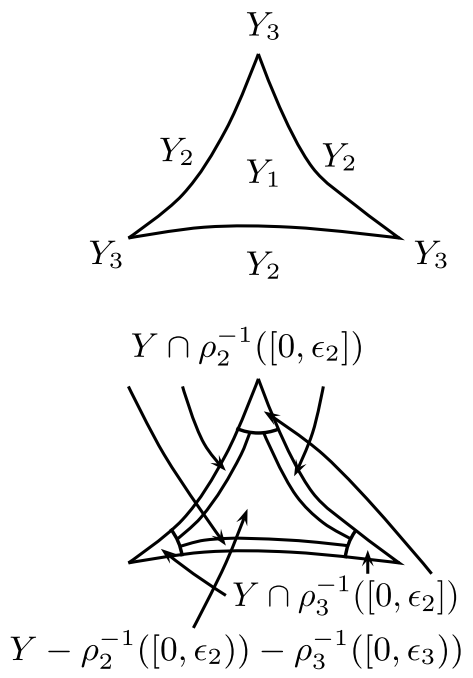

Figure 2

Whitney subanalytic analytic stratification does not necessarily satisfy the frontier condition, but the family of all connected components of the strata does so if the underlying set does not satisfy the closed condition, which we easily see (the upper panel of Figure 2).

Next, we consider stratifications of subanalytic maps. Let $\phi: X \rightarrow Y$ be a subanalytic map between subanalytic sets in $\mathbf{R}^{n}$. A subanalytic analytic stratification of $\phi$ is a pair consisting of subanalytic analytic stratifications $\left\{X_{i}\right\}_{i}$ of $X$ and $\left\{Y_{j}\right\}_{j}$ of $Y$ such that for each $i$, the restriction $\left.\phi\right|_{X_{i}}$ is an analytic submersion onto some $Y_{j}$. We write as $\phi:\left\{X_{i}\right\}_{i} \rightarrow\left\{Y_{j}\right\}_{j}$ and call it a subanalytic analytic stratified map. Assume that we have given subanalytic structures to $M, M / G$, and $\pi$. Then $\pi$ admits a subanalytic analytic stratification, as we will see. However, in general, a subanalytic analytic stratification of $\phi$ does not necessarily exist. For example, let $X=$ $\mathbf{N}$, let $Y=\mathbf{R}$, and define the map $\phi$ so that $\phi(k)=1 / k$ for $k \neq 0$. Then the map $\phi$ does not admit a subanalytic analytic stratification. Let us introduce a sufficient condition for the existence to explain the canonical subanalytic analytic stratification.

(*) The set $\phi^{-1}(B)$ is bounded in $\mathbf{R}^{n}$ for each bounded set $B$ in $\mathbf{R}^{n}$.

If condition $(*)$ is satisfied, then by $(9),(10)$, and (11) there always exists a subanalytic analytic stratification of $\phi$. Moreover, in the same way as 
for the canonical subanalytic analytic stratification of a subanalytic set, by replacing $\operatorname{Reg} X$ with $\operatorname{Reg} \phi$ we can construct a subanalytic analytic stratification of $\phi$ which has the following property and is called canonical. Let $A$ denote the family of subanalytic analytic stratifications of $\phi$. The canonical element in $A$ is the maximal one in the following partial order. Let $\phi:\left\{X_{i}\right\}_{i} \rightarrow\left\{Y_{j}\right\}_{j}$ and $\phi:\left\{X_{i^{\prime}}^{\prime}\right\}_{i^{\prime}} \rightarrow\left\{Y_{j^{\prime}}^{\prime}\right\}_{j^{\prime}}$ be elements of $A$. The former is smaller than the latter if the following conditions (15) and (16) and one of (17) and (18) are satisfied for some positive integer $k$ :

(15) $\left\{X_{i}: i, \operatorname{dim} \phi\left(X_{i}\right) \geq k+1\right\}=\left\{X_{i^{\prime}}^{\prime}: i^{\prime}, \operatorname{dim} \phi\left(X_{i^{\prime}}^{\prime}\right) \geq k+1\right\}$;

(16) $\left\{Y_{j}: j, \operatorname{dim} Y_{j} \geq k+1\right\}=\left\{Y_{j^{\prime}}^{\prime}: j^{\prime}, \operatorname{dim} Y_{j^{\prime}}^{\prime} \geq k+1\right\}$;

(17) $\left\{Y_{j}: j, \operatorname{dim} Y_{j}=k\right\} \neq\left\{Y_{j^{\prime}}^{\prime}: j^{\prime}, \operatorname{dim} Y_{j^{\prime}}^{\prime}=k\right\}$, and each $Y_{j}$ of dimension $k$ is included in some $Y_{j^{\prime}}^{\prime}$; and

(18) $\left\{Y_{j}: j, \operatorname{dim} Y_{j}=k\right\}=\left\{Y_{j^{\prime}}^{\prime}: j^{\prime}, \operatorname{dim} Y_{j^{\prime}}^{\prime}=k\right\},\left\{X_{i}: i, \operatorname{dim} \phi\left(X_{i}\right)=k\right\} \neq$ $\left\{X_{i^{\prime}}^{\prime}: i^{\prime}, \operatorname{dim} \phi\left(X_{i^{\prime}}^{\prime}\right)=k\right\}$, and each $X_{i}$ with $\operatorname{dim} g\left(X_{i}\right)=k$ is included in some $X_{i^{\prime}}^{\prime}$.

We call a subanalytic analytic stratified map $\phi:\left\{X_{i}\right\}_{i} \rightarrow\left\{Y_{j}\right\}_{j}$ a Whitney stratification of $\phi$ if the three stratifications $\left\{X_{i}\right\}_{i},\left\{Y_{j}\right\}_{j}$, and $\left\{\left.\operatorname{graph} \phi\right|_{X_{i}}\right\}_{i}$ (not only $\left\{X_{i}\right\}_{i}$ and $\left\{Y_{j}\right\}_{j}$ ) are Whitney stratifications. Under condition $(*)$, we can construct the canonical Whitney subanalytic analytic stratification of $\phi$ in the same way. The map $\pi: M \rightarrow M / G$ does not satisfy $(*)$ when it is not proper. However, we will construct the canonical Whitney subanalytic analytic stratification of the map $\pi: M \rightarrow M / G$ in exactly the same way. To prove Theorem 1.1, we will replace the map $\pi: M \rightarrow M / G$ with some subanalytic one $\phi: X \rightarrow Y$ and find the canonical Whitney stratification of $\phi$ but not a general Whitney stratification. The reason that we need the canonical one is that only the canonical one satisfies the conditions in Theorem 2.1 below.

If we consider all subanalytic maps, then notation becomes complicated. Hence, we add the following condition on maps. A analytic function on a subset $X \subset \mathbf{R}^{n}$ is the restriction to $X$ of a (real-valued) analytic function defined on an open neighborhood of $X$ in $\mathbf{R}^{n}$. An analytic map from a subset $X \subset \mathbf{R}^{n}$ to another $Y \subset \mathbf{R}^{n}$ is defined in the same way. Note that if $X$ is an analytic submanifold of $\mathbf{R}^{n}$, then this definition is equivalent to the one that the map is analytic in the usual sense. If the underlying map $\phi: X \rightarrow Y$ of an analytic stratified map $\phi:\left\{X_{i}\right\}_{i} \rightarrow\left\{Y_{j}\right\}_{j}$ is of analytic class, then the Whitney condition on $\left\{\text { graph }\left.\phi\right|_{X_{i}}\right\}_{i}$ in the definition of a Whitney stratification follows from the Whitney condition on $\left\{X_{i}\right\}_{i}$ and 
$\left\{Y_{j}\right\}_{j}$, where $X=\bigcup_{i} X_{i}$ and $Y=\bigcup_{j} Y_{j}$. We call $\phi:\left\{X_{i}\right\}_{i} \rightarrow\left\{Y_{j}\right\}_{j}$ an analytic stratified analytic map. (There exists a subanalytic analytic stratified (not analytic) map $\phi:\left\{X_{i}\right\}_{i} \rightarrow\left\{Y_{j}\right\}_{j}$ such that $\left\{X_{i}\right\}_{i}$ and $\left\{Y_{j}\right\}_{j}$ satisfy the Whitney condition but $\left\{\text { graph }\left.\phi\right|_{X_{i}}\right\}_{i}$ does not.) Moreover, in our arguments we replace the map $\phi: X \rightarrow Y$ with $\tilde{\phi}$, the restriction to the graph of $\phi$ of the projection $X \times Y \rightarrow Y$. Then the map $\tilde{\phi}: \operatorname{graph} \phi \rightarrow Y$ is of analytic class; $\tilde{\phi}$ is subanalytic if $\phi$ is so by (1) and the equality

$$
\begin{aligned}
\operatorname{graph} \tilde{\phi}= & \left\{(x, y, y) \in X \times Y^{2} \mid y=\phi(x)\right\} \\
= & \left\{\left(x, y, y^{\prime}\right) \in X \times Y^{2} \mid y=\phi(x)\right\} \\
& \cap\left\{\left(x, y, y^{\prime}\right) \in X \times Y^{2} \mid y^{\prime}=\phi(x)\right\} ;
\end{aligned}
$$

and graph $\phi$ satisfies the closed condition if $X$ does also. Hence, for simplicity of notation, we always consider (stratified) maps which are of analytic class.

Let $\left\{A_{\alpha}\right\}_{\alpha}$ and $\left\{B_{\beta}\right\}_{\beta}$ be families of subanalytic subsets of subanalytic sets $X, Y \subset \mathbf{R}^{n}$, respectively, finite locally at each point of $\mathbf{R}^{n}$. Then a subanalytic analytic stratification $\left\{X_{i}\right\}_{i}$ of $X$ is compatible with $\left\{A_{\alpha}\right\}_{\alpha}$ if each $A_{\alpha}$ is the union of some connected components of $X_{i}$. The canonical Whitney subanalytic analytic stratification $\left\{X_{i}\right\}_{i}$ of $X$ compatible with $\left\{A_{\alpha}\right\}_{\alpha}$ exists. Indeed, we define $X_{1}$ to be $\operatorname{Reg} X-\left\{x \in \overline{A_{\alpha}} \mid \operatorname{dim} A_{\alpha}<\operatorname{dim} X\right\}-\{x \in$ $\left.\overline{A_{\alpha}}-\operatorname{Reg} A_{\alpha} \mid \operatorname{dim} A_{\alpha}=\operatorname{dim} X\right\}$; we define $X_{2}$ by using $X-X_{1},\left\{A_{\alpha}-X_{1}\right\}_{\alpha}$ and the Whitney condition; and so on. Naturally, we also define a subanalytic analytic stratification of a subanalytic map $\phi: X \rightarrow Y$ compatible with $\left\{A_{\alpha}\right\}_{\alpha}$ and $\left\{B_{\beta}\right\}_{\beta}$. Under condition $(*)$, we can construct the canonical Whitney subanalytic analytic stratification $\phi:\left\{X_{j}\right\}_{j} \rightarrow\left\{Y_{k}\right\}_{k}$ of $\phi$ compatible with $\left\{A_{\alpha}\right\}_{\alpha}$ and $\left\{B_{\beta}\right\}_{\beta}$.

A Whitney subanalytic analytic stratified map $\phi:\left\{X_{i}\right\}_{i} \rightarrow\left\{Y_{j}\right\}_{j}$ or the underlying map $\phi: \bigcup_{i} X_{i} \rightarrow \bigcup_{j} Y_{j}$ is called a Thom map if the following condition is satisfied.

Let $X_{i}$ and $X_{i^{\prime}}$ be strata such that $\left(\overline{X_{i}}-X_{i}\right) \cap X_{i^{\prime}} \neq \emptyset$. If $\left\{a_{k}\right\}_{k}$ is a sequence of points of $X_{i}$ convergent to a point $b$ of $X_{i^{\prime}}$, and if the sequence of the tangent spaces $\left\{T_{a_{k}}\left(\left.\phi\right|_{X_{i}}\right)^{-1}\left(\phi\left(a_{k}\right)\right)\right\}_{k}$ converges to a space $T \subset \mathbf{R}^{n}$ in $G_{n, l}, l=\operatorname{dim}\left(\left.\phi\right|_{X_{i}}\right)^{-1}\left(\phi\left(a_{k}\right)\right)$, then $T_{b}\left(\left.\phi\right|_{X_{i^{\prime}}}\right)^{-1}(\phi(b)) \subset T$.

Note in the above condition that $\operatorname{dim}\left(\left.\phi\right|_{X_{i}}\right)^{-1}\left(\phi\left(a_{k}\right)\right) \geq \operatorname{dim}\left(\left.\phi\right|_{X_{i^{\prime}}}\right)^{-1}(\phi(b))$; that is, $\operatorname{dim} X_{i}-\operatorname{dim} \phi\left(X_{i}\right) \geq \operatorname{dim} X_{i^{\prime}}-\operatorname{dim} \phi\left(X_{i^{\prime}}\right)$; moreover, if $\left\{X_{i}\right\}_{i}$ and $\bigcup_{i} X_{i}$ satisfy the frontier condition and the closed condition, respectively, then $X_{i^{\prime}} \cap \bigcap_{l=1}^{\infty}\left(\bigcup_{k=l}^{\infty}\left(\left.\phi\right|_{X_{i}}\right)^{-1}\left(\phi\left(a_{k}\right)\right) \overline{)}=\left(\left.\phi\right|_{X_{i^{\prime}}}\right)^{-1}(\phi(b))\right.$, where the notation 
( ) denotes the closure of the set ( ). A typical example of a Thom map is as follows. Let $\phi: K \rightarrow L$ be a simplicial map between finite simplicial complexes, and embed $K$ and $L$ in $\mathbf{R}^{n}$. Then $\phi:\left\{\int \sigma \mid \sigma \in K\right\} \rightarrow\left\{\int \sigma \mid \sigma \in L\right\}$ is a Thom map. On the other hand, the restriction $\phi$ of the projection $\mathbf{R}^{3} \ni(x, y, z) \rightarrow(x, y) \in \mathbf{R}^{2}$ to the Whitney umbrella is an example where any Whitney subanalytic analytic stratification is not a Thom map because $\operatorname{dim} \phi^{-1}(\{(x, y)\})=1$ if $(x, y)=(0,0)$ and $\operatorname{dim} \phi^{-1}(\{(x, y)\})=0$ if $y \neq 0$ (see Figure 1). Consider the case where $\operatorname{dim} X_{i}-\operatorname{dim} \phi\left(X_{i}\right)$ is constant. (If $X$ and $Y$ are complex analytic manifolds, $\phi$ is complex analytic, and $\phi^{-1}(y)$ are of constant local dimension for all $y \in Y$, then $\phi$ is called flat.) In particular, let $X=[0, \infty) \times \mathbf{R}, Y=[0, \infty)$, let $\left\{X_{i}\right\}=\{\{0\} \times \mathbf{R},(0, \infty) \times \mathbf{R}\}$, let $\left\{Y_{j}\right\}=\{\{0\},(0, \infty)\}$, and let $\phi^{-1}(0)=\{0\} \times \mathbf{R}$. Then there is an ordinary differential equation $\frac{d x_{1}}{d x_{2}}=f\left(x_{1}, x_{2}\right)$ such that $\left\{\phi^{-1}(y) \mid y \in[0, \infty)\right\}$ is the integral curves of the equation, and $\phi:\left\{X_{i}\right\} \rightarrow\left\{Y_{j}\right\}$ is a Thom map if and only if $f$ is continuous. Figure 3 is the case of a Thom map, and Figure 4 is not the case of a Thom map. (There is a flat complex polynomial map between complex Euclidean spaces which is not locally triangulable; see [14, p. 311].)

It is easy to see that a proper Thom map is locally triangulable. Thom conjectured that a proper Thom map is globally triangulable (see [16]), and the second author proved the conjecture and its subanalytic version (see [15]). An example of a nonproper nontriangulable subanalytic Thom map is $\phi:\{X\} \rightarrow\{Y\}$, where $X=\mathbf{R}^{2}-\{(1 / n, n) \mid n \in \mathbf{N}, n>0\}, Y=\mathbf{R}$, and $\phi$ is the restriction to $X$ of the projection $\mathbf{R}^{2} \rightarrow \mathbf{R}$ to the former factor. However, this $\phi: X \rightarrow Y$ is subanalytically triangulable in the weak sense.

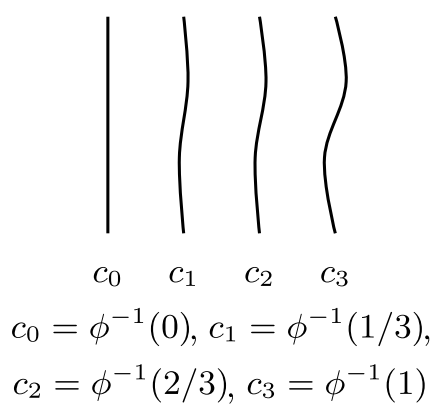

Figure 3 


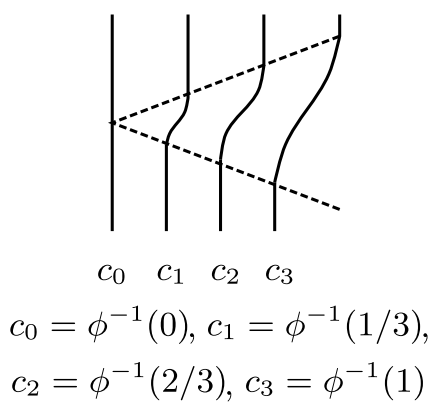

Figure 4

We do not know whether a general nonproper subanalytic Thom map is subanalytically triangulable in the weak sense.

We will use the following property of Thom maps. Let $\phi:\left\{X_{i}\right\}_{i} \rightarrow\left\{Y_{j}\right\}_{j}$ be a Thom map, let $U_{i}$ be a small open neighborhood of $X_{i}$ in the ambient Euclidean space, and let $\pi_{i}: U_{i} \rightarrow X_{i}$ be a submersive $C^{k}$ retraction. Then for each $X_{i^{\prime}}$ such that $\left(\overline{X_{i^{\prime}}}-X_{i^{\prime}}\right) \cap X_{i} \neq \emptyset$ and $y^{\prime} \in \phi\left(X_{i^{\prime}}\right)$, the restriction $\left.\pi_{i}\right|_{U_{i} \cap \phi^{-1}\left(y^{\prime}\right) \cap X_{i^{\prime}}}$ is a $C^{k}$ submersion into $\phi^{-1}(y) \cap X_{i}$ if $\pi_{i}\left(U_{i} \cap \phi^{-1}\left(y^{\prime}\right) \cap\right.$ $\left.X_{i^{\prime}}\right) \subset \phi^{-1}(y)$ for some $y \in \phi\left(X_{i}\right)$. We will look for such a map $\pi_{i}$.

Now, we remember tube systems. Let $m \geq 2$ be an integer. A subanalytic $C^{m}$ (not analytic) tube system $\left\{T_{j}=\left(\left|T_{j}\right|, \pi_{j}, \rho_{j}\right)\right\}_{j}$ for a Whitney subanalytic analytic stratification $\left\{Y_{j}\right\}_{j}$ of a subanalytic set $Y \subset \mathbf{R}^{n}$ consists of one tube $T_{j}$ at each $Y_{j}$, where $\pi_{j}:\left|T_{j}\right| \rightarrow Y_{j}$ is a subanalytic $C^{m}$ submersive retraction of an open subanalytic tubular neighborhood of $Y_{j}$ in $\mathbf{R}^{n}$, and $\rho_{j}$ is a nonnegative subanalytic $C^{m}$ function defined on $\left|T_{j}\right|$ such that $\rho_{j}^{-1}(0)=Y_{j}$ and each point $y$ of $Y_{j}$ is a unique and nondegenerate critical point of $\left.\rho_{j}\right|_{\pi_{j}^{-1}(y)}$. An example of a tube at $Y_{j}$ is a triple of a subanalytic tubular neighborhood of $Y_{j}$ in $\mathbf{R}^{n}$, the orthogonal projection from the neighborhood to $Y_{j}$, and the square of the function measuring the distance from $Y_{j}$. We call this tube standard. We can regard any tube as a standard one up to $C^{m}$ diffeomorphisms. To be precise, for any subanalytic $C^{m}$ tube $T_{j}=\left(\left|T_{j}\right|, \pi_{j}, \rho_{j}\right)$ at $Y_{j}$, there exists a subanalytic $C^{m}$ embedding $\xi_{j}$ of a subanalytic neighborhood of $Y_{j}$ in $\left|T_{j}\right|$ into $\mathbf{R}^{n}$ such that $\xi_{j}=\mathrm{id}$ on $Y_{j}$ and $\left(\operatorname{Im} \xi_{j}, \pi_{j} \circ \xi_{j}^{-1}, \rho_{j} \circ \xi_{j}^{-1}\right)$ is a standard tube (see [14, Lemma I.1.1]). A standard tube is of analytic class. However, we cannot choose standard tubes 
alone because we need the condition below on controlledness, and there does not necessarily exist a controlled tube system.

We call a tube system $\left\{T_{j}\right\}_{j}$ controlled if for each pair $j$ and $j^{\prime}$ such that $\operatorname{dim} Y_{j}<\operatorname{dim} Y_{j^{\prime}}$,

$$
\pi_{j} \circ \pi_{j^{\prime}}=\pi_{j} \quad \text { and } \quad \rho_{j} \circ \pi_{j^{\prime}}=\rho_{j} \quad \text { on }\left|T_{j}\right| \cap\left|T_{j^{\prime}}\right|,
$$

and we call a controlled tube system strongly controlled if (sc) the map $\left(\pi_{j}, \rho_{j}\right)_{Y_{j^{\prime}} \cap\left|T_{j}\right|}$ is a $C^{m}$ submersion into $Y_{j} \times \mathbf{R}$.

Note that condition (sc) follows from condition (ct) since $\left.\left(\pi_{j}, \rho_{j}\right)\right|_{Y_{j^{\prime}} \cap\left|T_{j}\right|^{\circ}}$ $\pi_{j^{\prime}}=\left(\pi_{j}, \rho_{j}\right)$ on $\left|T_{j}\right| \cap\left|T_{j^{\prime}}\right|$; hence, the definition of strongly controlledness coincides with the one of controlledness, and any Whitney subanalytic analytic stratification admits a strongly controlled subanalytic $C^{m}$ tube system (see [14, Lemma I.1.3]). The reason that we consider condition (sc) and use the term strongly controlled will become clear. The notation of a controlled tube system was introduced to show some properties of semialgebraic sets. The most important property shown by a controlled tube system is that for a semialgebraic and more generally subanalytic set $X$ in $\mathbf{R}^{n}$ and each point $x_{0}$ of $\mathbf{R}^{n}$, there exists $\epsilon>0$ such that the $\epsilon$-neighborhood of $x_{0}$ in $X \cup\left\{x_{0}\right\}$ is homeomorphic to the cone with vertex $x_{0}$ and base $\left\{x \in X \mid \operatorname{dis}\left(x, x_{0}\right)=\epsilon\right\}$. It may be possible to find analytic $\left\{T_{j}\right\}_{j}$, but it is difficult because the problem is to solve the analytic equations (ct).

In the proof of Lemmas 3.6' and 3.7-key lemmas of Theorem 1.1-we use the same idea as of the construction of $\left\{T_{j}\right\}_{j}$ satisfying condition (ct). Hence, we show the idea. Assume that the map $\pi_{j}$ and the function $\rho_{j}$ are given. Then by easy calculations, shrinking $\left|T_{j}\right|$ and $\left|T_{j^{\prime}}\right|$, we see that for each $x \in\left|T_{j}\right| \cap\left|T_{j^{\prime}}\right|$, the set $X_{j^{\prime}} \cap \pi_{j}^{-1}\left(\pi_{j}(x)\right) \cap \rho_{j}^{-1}\left(\rho_{j}(x)\right)$ is a subanalytic $C^{m}$ manifold, and we can define the value $\pi_{j^{\prime}}(x)$ to be the image of $x$ under the orthogonal projection to the manifold, though $\pi_{j^{\prime}}$ becomes of class $C^{m-1}$. Note that the family $\left\{X_{j^{\prime}} \cap \pi_{j}^{-1}\left(\pi_{j}(x)\right) \cap \rho_{j}^{-1}\left(\rho_{j}(x)\right)\left|x \in X_{j^{\prime}} \cap\right| T_{j} \mid\right\}$ is a subanalytic $C^{m}$ foliation of $X_{j^{\prime}} \cap\left|T_{j}\right|$.

We explain subanalytic triangulations of subanalytic sets constructed by the Whitney condition and controlled tube systems. This method is different from the one of Łojasiewicz in [7, Theorem 1]. Let $\left\{Y_{j}\right\}_{j}$ be a Whitney subanalytic analytic stratification of a subanalytic set $Y$ satisfying the closed condition, and let $\left\{T_{j}=\left(\left|T_{j}\right|, \pi_{j}, \rho_{j}\right)\right\}_{j}$ be a controlled subanalytic $C^{m}$ tube system for $\left\{Y_{j}\right\}_{j}$ such that $\operatorname{dim} Y_{1} \geq \operatorname{dim} Y_{2} \geq \cdots$. Each $Y_{j}$ has good properties as a manifold. However, the relation of $Y_{j}$ and $Y_{j+1} \cup Y_{j+2} \cup \cdots$ is not 
obvious. Hence, we separate $Y_{j}$ into $\bigcup_{j^{\prime}>j}\left\{y \in Y_{j} \cap\left|T_{j^{\prime}}\right| \mid \rho_{j^{\prime}}(y)<\epsilon_{j^{\prime}} \circ \pi_{j^{\prime}}(y)\right\}$ and its complement in $Y_{j}$, where $\epsilon_{j^{\prime}}$ is a small positive subanalytic analytic function on each $Y_{j^{\prime}}$ (see [14, Section II.5] for the topology of the space of subanalytic $C^{m}$ functions). Then by the Whitney condition, the latter set is a subanalytic $C^{m}$ manifold possibly with corners and satisfies the closed condition, and $Y_{j+1} \cup Y_{j+2} \cup \cdots$ is a subanalytic deformation retract of the union of $Y_{j+1} \cup Y_{j+2} \cup \cdots$ and the former set. We obtain subanalytic triangulations of the latter sets by the Cairns-Whitehead theorem and can canonically extend the triangulation to a subanalytic triangulation of $Y_{j} \cup Y_{j+1} \cup \cdots$ by the deformation retractions and condition (ct). Here we do not give the definition of the canonical extension and entrust it to [15]. We more precisely explain the separation as follows.

Let $\left\{Y_{j}\right\}_{j}=\left\{Y_{1}, \ldots, Y_{k}\right\}$. Assume that $Y$ is compact because the separation is clear in the compact case. By the Whitney condition we obtain a positive number $\delta_{k}$ and positive subanalytic functions $\delta_{i}: \mathbf{R}^{k-i} \rightarrow \mathbf{R}$, $i=k-1, \ldots, 2$, which satisfy the following conditions. Let $1 \leq l \leq i \leq k$ be integers, and let $\epsilon_{k}, \ldots, \epsilon_{2}$ be positive numbers satisfying $\epsilon_{k} \leq \delta_{k}, \epsilon_{k-1} \leq$ $\delta_{k-1}\left(\epsilon_{k}\right), \ldots, \epsilon_{2} \leq \delta_{2}\left(\epsilon_{k}, \ldots, \epsilon_{3}\right)$. Then the set

$$
E(i+1)=\mathbf{R}^{n}-\rho_{k}^{-1}\left(\left[0, \epsilon_{k}\right)\right)-\cdots-\rho_{i+1}^{-1}\left(\left[0, \epsilon_{i+1}\right)\right)
$$

is a subanalytic $C^{m}$ submanifold of $\mathbf{R}^{n}$ possibly with corners satisfying the closed condition, the three sets $E(i+1), \rho_{i}^{-1}\left(\epsilon_{i}\right)$, and $Y_{l}$ are transversals of each other, and the restrictions of $\rho_{i}$ to the sets $\rho_{i}^{-1}\left(\left(0, \epsilon_{i}\right]\right) \cap E(i+1)$ and $\rho_{i}^{-1}\left(\left(0, \epsilon_{i}\right]\right) \cap E(i+1) \cap Y_{l}$ are $C^{m}$ regular. We call such $\delta=\left\{\delta_{i}\right\}_{i=k, \ldots, 2}$ or $\epsilon=\left\{\epsilon_{i}\right\}_{i=k, \ldots, 2}$ removal data of $\left\{Y_{i}\right\}_{i=1, \ldots, k}$ (see Figure 2).

Assume that $Y$ is not compact and satisfies the closed condition. By the Whitney condition we have a positive proper subanalytic $C^{m}$ function $f$ on $\mathbf{R}^{n}$ such that $I$ - which denotes the common $C^{m}$ regular values of $f$, $\left.f\right|_{Y_{1}}, \ldots,\left.f\right|_{Y_{k}}$ is not bounded to the above. (If $\operatorname{dim} Y_{1}=0$, we do not call a point of $f\left(Y_{1}\right)$ a $C^{m}$ regular value.) Let $I^{\prime}$ be a subset of $I$ which is not bounded to the above and a finite union of closed intervals locally at each point of $\mathbf{R}$, and let $F$ denote the family of positive subanalytic $C^{m}$ functions on $\mathbf{R}$ which are locally constant on $\mathbf{R}-I^{\prime}$. Then there exist as in the compact case an element $\delta_{k} \in F$ and maps $\delta_{i}: F^{k-i} \rightarrow F, i=k-1, \ldots, 2$, with the following properties. Let $1 \leq l \leq i \leq k$ be integers, let $t \in I^{\prime}$, and let $\epsilon_{k}, \ldots, \epsilon_{2}$ be elements of $F$ satisfying $\epsilon_{k} \leq \delta_{k}, \epsilon_{k-1} \leq \delta_{k-1}\left(\epsilon_{k}\right), \ldots, \epsilon_{2} \leq \delta_{2}\left(\epsilon_{k}, \ldots, \epsilon_{3}\right)$. 
Set

$$
E(i+1)=\mathbf{R}^{n}-\bigcup_{j=k}^{i+1}\left\{x \in\left|T_{j}\right| \mid \rho_{j}(x)<\epsilon_{j} \circ f(x)\right\} .
$$

Then $E(i+1)$ is a subanalytic $C^{m}$ submanifold of $\mathbf{R}^{n}$ possibly with corners satisfying the closed condition; the four sets $E(i+1), f^{-1}(t),\left\{\rho_{i}(x)=\epsilon_{i}\right.$ 。 $f(x)\}$, and $Y_{l}$ are transversals of each other; and the restrictions of $\rho_{i}$ to the four sets $\left\{0<\rho_{i}(x) \leq \epsilon_{i} \circ f(x)\right\} \cap E(i+1),\left\{0<\rho_{i}(x) \leq \epsilon_{i} \circ f(x)\right\} \cap$ $E(i+1) \cap Y_{l},\left\{f(x)=t, 0<\rho_{i} \leq \epsilon_{i} \circ f(x)\right\} \cap E(i+1)$, and $\{f(x)=t, 0<$ $\left.\rho_{i}(x) \leq \epsilon_{i} \circ f(x)\right\} \cap E(i+1) \cap Y_{l}$ are $C^{m}$ regular. In this case too, we call $\delta=\left\{\delta_{i}\right\}_{i=k, \ldots, 2}$ or $\epsilon=\left\{\epsilon_{i}\right\}_{i=k, \ldots, 2}$ removal data of $\left\{Y_{i}\right\}_{i=1, \ldots, k}$. We separate each $Y_{j}$ into $Y_{j} \cap E(j+1)$ and $Y_{j}-E(j+1)$ in any case.

For a subanalytic analytic stratified map $\phi:\left\{X_{i}\right\}_{i} \rightarrow\left\{Y_{j}\right\}_{j}$, we need to consider two tube systems for $\left\{X_{i}\right\}_{i}$ and $\left\{Y_{j}\right\}_{j}$. Without loss of generality, let a Whitney subanalytic analytic stratification be of the form $\phi:\left\{X_{i, j}\right\}_{\substack{j=1, \ldots, k \\ i=1, \ldots, l_{j}}} \rightarrow\left\{Y_{j}\right\}_{j=1, \ldots, k}$ such that $\phi^{-1}\left(Y_{j}\right)=\bigcup_{i=1}^{l_{j}} X_{i, j}$ for each $j$. Set $X=\bigcup_{i} X_{i}$ and $Y=\bigcup_{j} Y_{j}$, and assume that $X, Y \subset \mathbf{R}^{n}$. Let $\left\{T_{j}=\right.$ $\left.\left(\left|T_{j}\right|, \pi_{j}, \rho_{j}\right)\right\}_{j}$ be a strongly controlled subanalytic $C^{m}$ tube system for $\left\{Y_{j}\right\}_{j}$, and let $\left\{T_{i, j}=\left(\left|T_{i, j}\right|, \pi_{i, j}, \rho_{i, j}\right)\right\}_{i, j}$ be a subanalytic $C^{m}$ tube system for $\left\{X_{i, j}\right\}_{i, j}$. We call $\left\{T_{i, j}\right\}_{i, j}$ controlled over $\left\{T_{j}\right\}_{j}$ if the following three conditions are satisfied.

(sc1) For each $(i, j), \tilde{\phi}\left(\left|T_{i, j}\right|\right) \subset\left|T_{j}\right|$ and $\phi \circ \pi_{i, j}=\pi_{j} \circ \tilde{\phi}$ on $\left|T_{i, j}\right|$, where $\tilde{\phi}$ is a subanalytic analytic extension of $\phi$ to a subanalytic open neighborhood of $X$ in $\mathbf{R}^{n}$.

(sc2) For each $j,\left\{T_{i, j}\right\}_{i=1, \ldots, l_{j}}$ is a strongly controlled tube system for $\left\{X_{i, j}\right\}_{i=1, \ldots, l_{j}}$.

$(\operatorname{sc} 3)^{\prime}$ For any pair $(i, j)$ and $\left(i^{\prime}, j^{\prime}\right)$ such that $\operatorname{dim} X_{i, j}<\operatorname{dim} X_{i^{\prime}, j^{\prime}}$, it holds that $\pi_{i, j} \circ \pi_{i^{\prime}, j^{\prime}}=\pi_{i, j}$ on $\left|T_{i, j}\right| \cap\left|T_{i^{\prime}, j^{\prime}}\right|$.

Moreover, if the map $\left(\pi_{i, j}, \phi\right)_{X_{i^{\prime}, j^{\prime}} \cap\left|T_{i, j}\right|}$ in $(\mathrm{sc} 3)^{\prime}$ is a $C^{m}$ submersion into the $C^{m}$ manifold $\left\{(x, y) \in X_{i, j} \times\left(Y_{j^{\prime}} \cap\left|T_{j}\right|\right) \mid \phi(x)=\pi_{j}(y)\right\}$, then $\left\{T_{i, j}\right\}_{i, j}$ is called strongly controlled over $\left\{T_{j}\right\}_{j}$, and condition $(\mathrm{sc} 3)^{\prime}$ together with this condition is called ( $\mathrm{sc} 3)$. Here ( $\mathrm{sc} 3)^{\prime}$ does not necessarily imply (sc3); that is, we can construct a stratified map $\phi:\left\{X_{i, j}\right\}_{i, j} \rightarrow\left\{Y_{j}\right\}_{j}$ so that there exist controlled $\left\{T_{j}\right\}_{j}$ and $\left\{T_{i, j}\right\}_{i, j}$ controlled over $\left\{T_{j}\right\}_{j}$ but there does not exist controlled $\left\{T_{j}\right\}_{j}$ and $\left\{T_{i, j}\right\}_{i, j}$ strongly controlled over $\left\{T_{j}\right\}_{j}$.

It is natural to require the following simple condition in place of conditions (sc2) and (sc3). 
(sc4) The tube system $\left\{T_{i, j}\right\}_{i, j}$ is strongly controlled.

However, this condition cannot be necessarily satisfied. An example is given as follows. Define the following:

$$
\begin{aligned}
Y & =R^{2}, \quad Y_{3}=\{0\}, \quad Y_{2}=(\mathbf{R} \times\{0\}) \cup(\{0\} \times \mathbf{R})-\{0\}, \\
Y_{1} & =Y-Y_{2}-Y_{3}, \\
\psi(x, y) & =|x|^{1 / 2}+|y|^{1 / 2} \quad \text { for }(x, y) \in \mathbf{R}^{2}, \\
X & =\operatorname{graph} \psi, \quad X_{1, j}=\left.\operatorname{graph} \psi\right|_{Y_{j}}, \quad j=1,2,3, \\
\phi(x, y, z) & =(x, y) \quad \text { for }(x, y, z) \in X,
\end{aligned}
$$

and $\left\{T_{j}\right\}_{j=2,3}$ a strongly controlled subanalytic $C^{m}$ tube system for $\left\{Y_{j}\right\}_{j=2,3}$.

Then there does not exist a strongly controlled subanalytic $C^{m}$ tube system $\left\{T_{1, j}\right\}_{j=2,3}$ for $\left\{X_{1, j}\right\}_{j=2,3}$ satisfying condition (sc1). Contrarily, assume that such a one exists. Without loss of generality, we can suppose that $T_{2}$ is standard and hence that $\pi_{2}(x, y)=x$ on a neighborhood of $(0, \infty) \times\{0\}$. Then by condition ( $\mathrm{sc} 1)$,

$$
\pi_{1,2}\left(x, y,|x|^{1 / 2}+|y|^{1 / 2}\right)=\left(x, 0,|x|^{1 / 2}\right) \quad \text { for }(x, y) \in Y_{1} \text { near }(0, \infty) \times\{0\}
$$

Hence, for each $x \in \mathbf{R}$, the tangent line of the curve $\pi_{1,2}^{-1}\left(\left\{\left(x, 0,|x|^{1 / 2}\right)\right\}\right) \cap$ $X$ at $\left(x, 0,|x|^{1 / 2}\right)$ is $\{0\} \times \mathbf{R}$, and the curve is included in the 2-manifold $\rho_{1,3}^{-1}\left(\rho_{1,3}\left(x, 0,|x|^{1 / 2}\right)\right)$ if $x$ is close to 0 . Therefore, the angle formed by the tangent line at $\left(x, 0,|x|^{1 / 2}\right)$ and the line $\mathbf{R}\left(x, 0,|x|^{1 / 2}\right)$ converges to 0 as $x \rightarrow 0$. However, some $C^{m}$ diffeomorphism carries the 2-manifolds $\rho_{1,3}^{-1}(a)$ (with $a$ small positive numbers) to the spheres $\left\{(x, y, z) \in \mathbf{R}^{3} \mid x^{2}+y^{2}+z^{2}=\right.$ $a\}$, and hence, for a point $b$ in $\mathbf{R}^{3}-\{0\}$ near 0 and a nonzero tangent vector $v$ of $\rho_{1,3}^{-1}\left(\rho_{1,3}(b)\right)$ at $b$, the angle $\angle(\mathbf{R} b, \mathbf{R} v)$ does not converge to 0 as $b \rightarrow 0$. That is a contradiction. Note that $\phi:\left\{X_{1, j}\right\}_{j} \rightarrow\left\{Y_{j}\right\}_{j}$ is a Thom map.

By conditions ( $\mathrm{sc} 1)$ and $(\mathrm{sc} 3)$, the maps $\pi_{i, j}$ are useful. On the other hand, the functions $\rho_{i, j}$ are not useful because condition (sc4) fails, and we will treat only the case where the set of indices of $X_{i, j}$ is a singleton for each fixed $j$ and hence condition (sc2) is automatically satisfied. We use $\rho_{j} \circ \tilde{\phi}$ in place of $\rho_{i, j}$, and we write $\rho_{i, j}$ for form's sake in the following arguments.

The Thom conjecture in the subanalytic case was proved as follows. It is easy to see that if $\left\{T_{i, j}\right\}_{i, j}$ is controlled over $\left\{T_{j}\right\}_{j}$ and $\phi:\left\{X_{i, j}\right\}_{i, j} \rightarrow\left\{Y_{j}\right\}_{j}$ is a Thom map, then condition ( $\mathrm{sc} 3)$ is satisfied, that is, that $\left\{T_{i, j}\right\}_{i, j}$ is 
strongly controlled over $\left\{T_{j}\right\}_{j}$. Moreover, for a Thom map $\phi:\left\{X_{i, j}\right\}_{i, j} \rightarrow$ $\left\{Y_{j}\right\}_{j}$ and a strongly controlled subanalytic $C^{m}$ tube system $\left\{T_{j}\right\}_{j}$ for $\left\{Y_{j}\right\}_{j}$, there exists a subanalytic $C^{m}$ tube system $\left\{T_{i, j}\right\}_{i, j}$ for $\left\{X_{i, j}\right\}_{i, j}$ controlled over $\left\{T_{j}\right\}_{j}$ if the underlying map $\phi: X \rightarrow Y$ is of analytic class (see [14, Lemma I.1.3 $\left.\left.3^{\prime}\right]\right)$. Then for $X_{i, j}$ and $X_{i^{\prime}, j^{\prime}}$ with $X_{i, j} \cap\left(\overline{X_{i^{\prime}, j^{\prime}}}-X_{i^{\prime}, j^{\prime}}\right) \neq \emptyset$ and $Y_{j} \cap\left(\overline{Y_{j^{\prime}}}-Y_{j^{\prime}}\right) \neq \emptyset$ and points $x_{i, j}$ in $X_{i, j}$ and $x_{i^{\prime}, j^{\prime}}$ in $X_{i^{\prime}, j^{\prime}}$ close to $x_{i, j}$ such that $\pi_{i, j}\left(x_{i^{\prime}, j^{\prime}}\right)=x_{i, j}$, the germ of the restriction $\left.\pi_{i, j}\right|_{\phi^{-1}\left(\phi\left(x_{i^{\prime}, j^{\prime}}\right)\right) \cap X_{i^{\prime}, j^{\prime}}}$ at $x_{i^{\prime}, j^{\prime}}$ is a $C^{k}$ submersion into $\phi^{-1}\left(\phi\left(x_{i, j}\right)\right) \cap X_{i, j}$. By this property it was shown in [15, Theorem] that if a proper subanalytic analytic map $\phi: X \rightarrow Y$ admits a Whitney subanalytic analytic stratification $\phi:\left\{X_{i, j}\right\}_{i, j} \rightarrow\left\{Y_{j}\right\}_{j}$, a strongly controlled subanalytic $C^{m}$ tube system $\left\{T_{j}\right\}_{j}$ for $\left\{Y_{j}\right\}_{j}$, and a subanalytic $C^{m}$ tube system $\left\{T_{i, j}\right\}_{i, j}$ for $\left\{X_{i, j}\right\}_{i, j}$ strongly controlled over $\left\{T_{j}\right\}_{j}$, then the map $\phi$ has a subanalytic triangulation. Here the assumption that the map $\phi$ is proper is too strong to apply to the case of $\pi: M \rightarrow M / G$. We replace it with the two conditions that the set of indexes of $\left\{X_{i, j}\right\}_{i, j}$ is the same as that of $\left\{Y_{j}\right\}_{j}$, that is, that $\left\{X_{i, j}\right\}_{i}$ consists of one element for each $j$ and the map $\left.\left(\pi_{i, j}, \phi\right)\right|_{X \cap\left|T_{i, j}\right|}: X \cap\left|T_{i, j}\right| \rightarrow X_{j} \times\left|T_{j}\right|$ is proper, which are satisfied by the map $\pi: M \rightarrow M / G$. Then we have the following.

TheOREm 2.1. Let $m \geq 2$ be an integer. Let $\phi: X \rightarrow Y$ be a subanalytic analytic map, let $\phi:\left\{X_{j}\right\}_{j} \rightarrow\left\{Y_{j}\right\}_{j}$ be its Whitney subanalytic analytic stratification such that $X_{j}=\phi^{-1}\left(Y_{j}\right)$ for each $j$, let $\left\{T_{Y, j}=\left(\left|T_{Y, j}\right|\right.\right.$, $\left.\left.\pi_{Y, j}, \rho_{Y, j}\right)\right\}_{j}$ be a strongly controlled subanalytic $C^{m}$ tube system for $\left\{Y_{j}\right\}_{j}$, and let $\left\{T_{X, j}=\left(\left|T_{X, j}\right|, \pi_{X, j}, \rho_{X, j}\right)\right\}_{j}$ be a subanalytic $C^{m}$ tube system for $\left\{X_{j}\right\}_{j}$ strongly controlled over $\left\{T_{Y, j}\right\}_{j}$. Assume that the map $\left.\left(\pi_{X, j}, \phi\right)\right|_{X \cap\left|T_{X, j}\right|}$ : $X \cap\left|T_{X, j}\right| \rightarrow X_{j} \times\left|T_{Y, j}\right|$ is proper for each $j$. Then the map $\phi$ admits a subanalytic triangulation.

Sketch of proof of Theorem 2.1. For the time being, we assume that the map $\phi$ is proper. Let us recall the proof in [15, Theorem]. We proceed as in the above triangulations of subanalytic sets (not maps). We separate $X_{j}$ and $Y_{j}$ by $\rho_{Y, j^{\prime}} \circ \tilde{\phi}$ and $\rho_{Y, j^{\prime}}$, respectively, as we have explained. We order the set of indexes so that $\operatorname{dim} Y_{j}>\operatorname{dim} Y_{j+1}$. For removal data $\left\{\epsilon_{j}\right\}_{j}$ of $\left\{T_{Y, j}\right\}_{j}$, set

$$
\begin{aligned}
& \hat{X}_{j}=X-\bigcup_{i>j}\left\{x \in\left|T_{X, i}\right| \mid \rho_{Y, i} \circ \tilde{\phi}(x)<\epsilon_{i} \circ \phi \circ \pi_{X, i}(x)\right\}, \\
& \hat{Y}_{j}=Y-\bigcup_{i>j}\left\{y \in\left|T_{Y, i}\right| \mid \rho_{Y, i}(y)<\epsilon_{i} \circ \pi_{Y, i}(y)\right\}
\end{aligned}
$$

(see Figure 5). 


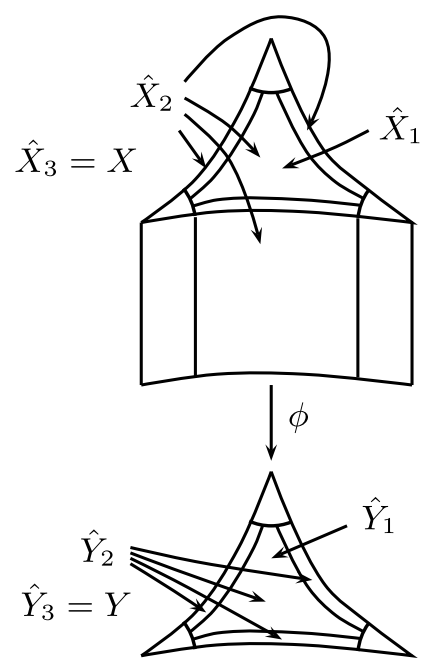

Figure 5

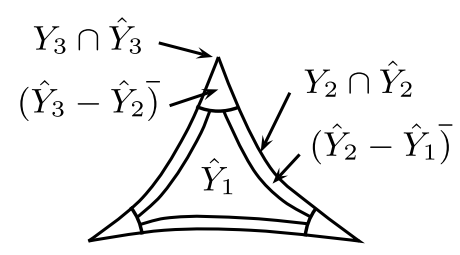

Figure 6

Then $Y_{j} \cap \hat{Y}_{j}$ is a subanalytic $C^{m}$ submanifold of $Y_{j}$ possibly with corners satisfying the closed condition for each $j$, and by the strongly controlledness of $\left\{T_{Y, j}\right\}_{j}$, there is a subanalytic homotopy $\gamma_{Y, j+1, t}, 0 \leq t \leq 1$, of $\left(\hat{Y}_{j+1}-\hat{Y}_{j}\right)$ of a deformation retraction from $\left(\hat{Y}_{j+1}-\hat{Y}_{j}\right)$ to $Y_{j+1} \cap \hat{Y}_{j+1}$ such that $\gamma_{Y, j+1,0}=\mathrm{id}, \gamma_{Y, j+1, t}=\mathrm{id}$ on $Y_{j+1} \cap \hat{Y}_{j+1}, \pi_{Y, j+1} \circ \gamma_{Y, j+1, t}=\pi_{Y, j+1}$, and $\rho_{Y, j+1} \circ \gamma_{Y, j+1, t}=(1-t) \rho_{Y, j+1}$; hence, $\gamma_{Y, j+1,1}=\pi_{Y, j+1}, \gamma_{Y, j+1, t}$ is a homeomorphism onto its image for each $t \neq 1$, and $\gamma_{Y, j+1, t}$ preserves the sets

$$
\left(\hat{Y}_{j+1}-\hat{Y}_{j} \overline{)} \cap\left\{y \in\left|T_{Y, j}\right| \mid \rho_{Y, i}(y)=\epsilon_{i} \circ \pi_{Y, i}(y)\right\}, \quad i=j+2, \ldots\right.
$$

(see Figure 6). Here existence of a (not necessarily subanalytic) homotopy $\gamma_{Y, j+1, t}$ follows from the usual arguments of differential topology (see [2, 
Chapter II]). However, for subanalytic $\gamma_{Y, j+1, t}$ we need a theory of subanalytic sets and maps (see [14, Section II.6]).

By properness of the map $\phi$, we can choose the removal data so small that each $\hat{X}_{j}$ is a closed subset of $X$. Then by the strongly controlledness of $\left\{T_{X, j}\right\}_{j}$ over $\left\{T_{Y, j}\right\}_{j}, X_{j} \cap \hat{X}_{j}$ is also a subanalytic $C^{m}$ submanifold of $X_{j}$ possibly with corners satisfying the closed condition; the map $\left.\phi\right|_{X_{j} \cap \hat{X}_{j}}: X_{j} \cap \hat{X}_{j} \rightarrow Y_{j} \cap \hat{Y}_{j}$ is a subanalytic $C^{m}$ fiber bundle over each connected component of $Y_{j} \cap \hat{Y}_{j}$ and carries $\partial\left(X_{j} \cap \hat{X}_{j}\right)$ to $\partial\left(Y_{j} \cap \hat{Y}_{j}\right)$; and there is a subanalytic homotopy $\gamma_{X, j+1, t}, 0 \leq t \leq 1$, of $\left(\hat{X}_{j+1}-\hat{X}_{j}\right)$ which satisfies the same conditions as $\gamma_{Y, j+1, t}$ and the equality $\gamma_{Y, j+1, t} \circ \phi=\phi \circ \gamma_{X, j+1, t}$.

We construct a subanalytic triangulation of the restriction $\left.\phi\right|_{\hat{X}_{j}}: \hat{X}_{j} \rightarrow Y_{j}$ by induction on $j$. First, by the Cairns-Whitehead theorem we obtain a subanalytic $C^{m}$ triangulation $\left(\tau_{X, 1}: P_{X, 1} \rightarrow X_{1} \cap \hat{X}_{1}, \tau_{Y, 1}: P_{Y, 1} \rightarrow Y_{1} \cap \hat{Y}_{1}\right)$ of the map $\left.\phi\right|_{X_{1} \cap \hat{X}_{1}}: X_{1} \cap \hat{X}_{1} \rightarrow Y_{1} \cap \hat{Y}_{1}$. (Note that $X_{1} \cap \hat{X}_{1}=\hat{X}_{1}$ and $Y_{1} \cap \hat{Y}_{1}=\hat{Y}_{1}$.) By induction, we assume that there exists a subanalytic triangulation $\left(\tau_{X, j}: P_{X, j} \rightarrow \hat{X}_{j}, \tau_{Y, j}: P_{Y, j} \rightarrow \hat{Y}_{j}\right)$ of the map $\left.\phi\right|_{\hat{X}_{j}}: \hat{X}_{j} \rightarrow \hat{Y}_{j}$ for some $j \in \mathbf{N}$ such that the restriction $\left(\left.\tau_{X, j}\right|_{\tau_{X, j}^{-1}\left(X_{k} \cap \hat{X}_{k}\right)}: \tau_{X, j}^{-1}\left(X_{k} \cap \hat{X}_{k}\right) \rightarrow\right.$ $\left.X_{k} \cap \hat{X}_{k},\left.\tau_{Y, j}\right|_{\tau_{Y, j}^{-1}\left(Y_{k} \cap \hat{Y}_{k}\right)}: \tau_{Y, j}^{-1}\left(Y_{k} \cap \hat{Y}_{k}\right) \rightarrow Y_{k} \cap \hat{Y}_{k}\right)$ is a subanalytic $C^{m}$ triangulation of the map $\left.\phi\right|_{X_{k} \cap \hat{X}_{k}}: X_{k} \cap \hat{X}_{k} \rightarrow Y_{k} \cap \hat{Y}_{k}$ for each $k \leq j$. Then we canonically extend $\tau_{Y, j}$ to a subanalytic triangulation $\tau_{Y, j+1}: P_{Y, j+1} \rightarrow \hat{Y}_{j+1}$ so that the restriction $\left.\tau_{Y, j+1}\right|_{\tau_{Y, j+1}^{-1}\left(Y_{j+1} \cap \hat{Y}_{j+1}\right)}: \tau_{Y, j+1}^{-1}\left(Y_{j+1} \cap \hat{Y}_{j+1}\right) \rightarrow Y_{j+1} \cap$ $\hat{Y}_{j+1}$ is a subanalytic $C^{m}$ triangulation of $Y_{j+1} \cap \hat{Y}_{j+1}$ by $\rho_{Y, j+1}, \pi_{Y, j+1}$, and $\gamma_{Y, j+1, t}$. Since the extension is canonical, we can lift, in the same canonical way, $\tau_{Y, j+1}$ to a subanalytic triangulation $\tau_{X, j+1}$ of $\hat{X}_{j+1}$ so that $\tau_{X, j+1}$ is an extension of $\tau_{X, j}$ and $\left(\tau_{X, j+1}, \tau_{Y, j+1}\right)$ is a subanalytic triangulation of the restriction $\left.\phi\right|_{\hat{X}_{j+1}}$ and has the above property of $\left(\tau_{X, j}, \tau_{Y, j}\right)$ by the strong controlledness of $\left\{T_{X, i}\right\}_{i}$ over $\left\{T_{Y, i}\right\}_{i}$ and $\gamma_{X, j+1, t}$. Thus, by induction we obtain a subanalytic triangulation of $\phi$.

If the map $\phi$ is not proper, then there is the case where the set $\pi_{X, j}^{-1}(x)$ becomes small as $X_{j} \ni x \rightarrow \infty$ (see Figure 7 ); the image of $\hat{X}_{1}$ under $\phi$ in Figure 7 is not included in $\hat{Y}_{1}$; the restriction $\left.\phi\right|_{\hat{X}_{1}}: \hat{X}_{1} \rightarrow Y_{1}$ is not a $C^{m}$ fiber bundle over each connected component of $\hat{Y}_{1}$; we cannot lift a triangulation of $\hat{Y}_{1}$ to one of $\hat{X}_{1}$; and hence, the above arguments do not work. However, if the map $\left.\left(\pi_{X, j+1}, \phi\right)\right|_{X \cap\left|T_{X, j+1}\right|}: X \cap\left|T_{X, j+1}\right| \rightarrow X_{j+1} \times\left|T_{Y, j+1}\right|$ is proper, then the restriction $\left.\phi\right|_{\hat{X}_{1}}: \hat{X}_{1} \rightarrow \hat{Y}_{1}$ is a subanalytic $C^{m}$ fiber bundle over 


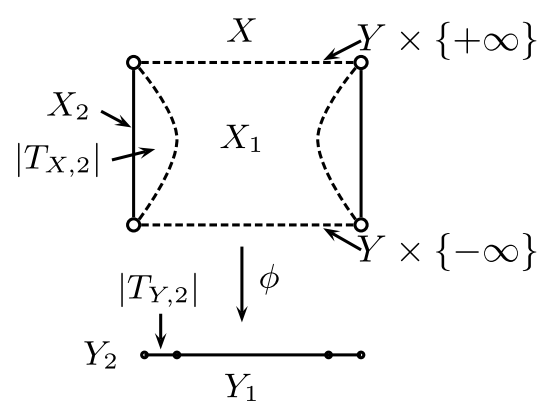

Figure 7

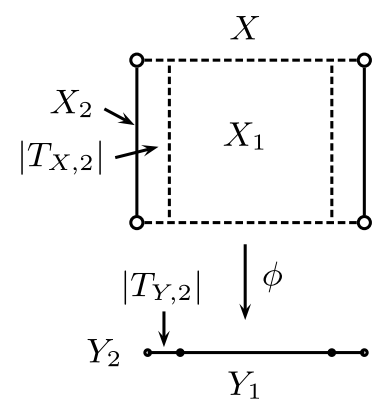

Figure 8

each connected component of $\hat{Y}_{1}$, for any $j$, too; $\left.\phi\right|_{X_{j} \cap \hat{X}_{j}}: X_{j} \cap \hat{X}_{j} \rightarrow Y_{j} \cap \hat{Y}_{j}$ has the same property; and the above arguments work (see Figure 8). Hence, Theorem 2.1 holds true. We omit the details.

\section{§3. Proof of Theorem 1.1}

In this section we prove Theorem 1.1. We modify the map $\pi: M \rightarrow M / G$ so that we can apply Theorem 2.1. Set $n=\operatorname{dim} M$. For $x \in M$, let $G_{x}$ denote the isotropy group at $x$ of $G$. A subset $S$ of $M$ containing $x$ is called a nearslice at $x$ if $G_{x} S=S$, and there exist an open neighborhood $U$ of $G_{x} / G_{x}$ in $G / G_{x}$ and a local cross section $\chi: U \rightarrow G$ of the map $G \rightarrow G / G_{x}$ such that the map $U \times S \ni(u, s) \rightarrow \chi(u) s \in M$ is a homeomorphism onto an open neighborhood of $x$ in $M$. Assume that $M$ is an analytic $G$-manifold. An analytic submanifold $S$ of $M$ containing $x$ is called a linear slice at $x$ if 
$G_{x} S=S$, the map $G \times{ }_{G_{x}} S \ni(g, s) \rightarrow g s \in M$ is an analytic diffeomorphism onto an open neighborhood of $x$ in $M$, and $S$ is $G_{x}$-equivariantly analytic diffeomorphic to a Euclidean space where $G_{x}$ acts orthogonally. Here $G \times{ }_{G_{x}}$ $S$ is the quotient space of $G \times S$ under the equivalence relation $\left(g g^{\prime}, s\right) \sim$ $\left(g, g^{\prime} s\right)$ for $\left(g, g^{\prime}, s\right) \in G \times G_{x} \times S$.

LEMma 3.1. We can assume in Theorem 1.1 that $M$ is a proper subanalytic analytic $G$-manifold and satisfies the closed condition.

Proof. Let $M$ be a proper (not subanalytic) $C^{k} G$-manifold. Assume that $1<k \leq \infty$. Then $M$ is equivariantly $C^{k}$ diffeomorphic to some analytic $G$ manifold. This was shown by Palais $[13$, Theorem B] in the case of compact $G$ and $M$, by Matumoto and Shiota [9, Theorem 1.3] in the case of compact $G$, and by Illman [5, Theorem A] in the general case.

Assume that $k=1$. Then there is only one problem in the proof in [5, Theorem A]. The following theorem of Koszul [6, p. 139] is used in $[5$, Theorem A].

FACT. If $X$ is a differentiable $G$-manifold and the isotropy group $G_{x}$ at a point $x \in X$ is compact, then there exists a near-slice at $x$.

The problem is that the proof of the fact in $[6$, p. 139] and $[12$, Theorem 2.3.3] works for $X$ of class $C^{k}, k>1$. Hence, we need to prove the fact in the $C^{1}$ case. Regard $X$ as a $C^{1} G_{x}$-manifold. Note that $G_{x}$ is a compact Lie group, which is an analytic submanifold of $G$. Then by [9, Theorem 1.3] there exist an analytic $G_{x}$-manifold $X^{*}$ and a $C^{1} G_{x^{-}}$equivariant diffeomorphism $f: X \rightarrow X^{*}$. Set $f(x)=x^{*}$, and set $f(G x)=Y$, which is a $C^{1}$ submanifold of $X^{*}$ and contains $x^{*}$. Choose an analytic Riemannian metric on $X^{*}$ invariant under $G_{x}$. Let $\epsilon>0$ be a small number, define an analytic submanifold $S^{*}$ of $X^{*}$ to be the union of geodesic segments of length smaller than $\epsilon$ starting from $x^{*}$ in a direction orthogonal to $T_{x^{*}} Y$ - the tangent space of $Y$ at $x^{*}$-and set $S=f^{-1}\left(S^{*}\right)$. Then $S$ together with some local cross section of the map $G \rightarrow G / G_{x}$ satisfies the conditions of a near-slice, and the fact is proved.

Thus, $M$ is equivariantly $C^{k}$ diffeomorphic to some analytic $G$-manifold $M^{\prime}$. Then we can replace $M$ with $M^{\prime}$ for the following reason. Let $f: M \rightarrow$ $M^{\prime}$ and $\bar{f}: M / G \rightarrow M^{\prime} / G$ be the diffeomorphism and the induced homeomorphism, respectively; assume that Theorem 1.1 is proved for $M^{\prime}$, and let $\left(\tau^{\prime}: P^{\prime} \rightarrow M^{\prime}, \sigma^{\prime}: L^{\prime} \rightarrow M^{\prime} / G\right)$ be a resulting triangulation of the natural map $\pi^{\prime}: M^{\prime} \rightarrow M^{\prime} / G$. Then $\left(f^{-1} \circ \tau^{\prime}: P^{\prime} \rightarrow M, \bar{f}^{-1} \circ \sigma^{\prime}: L^{\prime} \rightarrow M / G\right)$ is the 
required triangulation of the map $\pi: M \rightarrow M / G$. Here $M^{\prime}$ can be embedded in some Euclidean space as a closed subset. Hence, $M^{\prime}$ can be subanalytic and satisfies the closed condition.

Next, we consider the case where $M$ is a proper subanalytic $C^{k} G$ manifold. The above proof and the proof in [5] are valid in the subanalytic category. Hence, $M$ is equivariantly subanalytically $C^{k}$ diffeomorphic to a subanalytic analytic $G$-manifold $M^{\prime}$. Let $f: M \rightarrow M^{\prime}$ and $\bar{f}: M / G \rightarrow M^{\prime} / G$ denote the diffeomorphism and the homeomorphism, respectively; let $\pi^{\prime}$ : $M^{\prime} \rightarrow M^{\prime} / G$ be the natural map, and let $\left(\tau^{\prime}: P^{\prime} \rightarrow M^{\prime}, \sigma^{\prime}: L^{\prime} \rightarrow M^{\prime} / G\right)$ be a subanalytic triangulation of the map $\pi^{\prime}: M^{\prime} \rightarrow M^{\prime} / G$. Then it suffices to see that the maps $f^{-1} \circ \tau^{\prime}$ and $\bar{f} \circ \sigma^{\prime}$ are subanalytic. More generally, we see that the composite of two subanalytic maps is subanalytic if the first source space satisfies the closed condition. Let $f_{1}: X_{1} \rightarrow X_{2}$ and $f_{2}: X_{2} \rightarrow X_{3}$ be subanalytic maps such that $X_{1}$ satisfies the closed condition. Then the following set is subanalytic by the property (1) in Section 2:

$$
X_{4}=\left\{\left(x_{1}, x_{2}, x_{2}^{\prime}, x_{3}^{\prime}\right) \in \operatorname{graph} f_{1} \times \operatorname{graph} f_{2} \mid x_{2}=x_{2}^{\prime}\right\} ;
$$

the graph of $f_{2} \circ f_{1}$ is the image of $X_{4}$ under the projection $p: X_{1} \times X_{2} \times X_{2} \times$ $X_{3} \rightarrow X_{1} \times X_{3}$, the restriction $\left.p\right|_{X_{4}}$ is a homeomorphism onto graph $f_{2} \circ f_{1}$, and graph $f_{2} \circ f_{1}$ satisfies the closed condition. Hence, by (11), graph $f_{2} \circ f_{1}$ is subanalytic, and we can replace $M$ with $M^{\prime}$. Then we can assume that $M^{\prime}$ satisfies the closed condition as above. Thus, Lemma 3.1 is proved.

From now on, we assume that $M$ is a proper subanalytic analytic $G$ manifold satisfying the closed condition. We will give a subanalytic structure to $M / G$. A subanalytic $G$-set $X$ is a subanalytic set where $G$ acts so that the action $G \times X \rightarrow X$ is subanalytic and analytic. (Remember that the map is analytic if it is extended to an analytic map between open neighborhoods of $G \times X$ and $X$ in their ambient Euclidean spaces.) As we have noted, we can assume that $M \subset \mathbf{R}^{2 n+1}$. However, we regard $\mathbf{R}^{2 n+1}$ as a subanalytic $G$-set where $G$ trivially acts, and hence the inclusion map of $M$ into $\mathbf{R}^{2 n+1}$ is not a $G$-map.

Lemma 3.2. In Theorem 1.1 we can replace the set $M$, the set $M / G$, and the map $\pi: M \rightarrow M / G$ with a subanalytic $G$-set $X$ satisfying the closed condition, a subanalytic set $Y$ satisfying the closed condition, and a subanalytic analytic map $\phi: X \rightarrow Y$, respectively. (By this replacing we lose analyticity of $M$, keep subanalyticity of $M$ and analyticity of the action, and obtain analyticity and subanalyticity of the map $\phi$. 
Proof. By [8, Theorem 3.3] there exists a $G$-invariant subanalytic map $p: M \rightarrow \mathbf{R}^{2 n+1}$ such that $p(M)$ is closed and subanalytic in $\mathbf{R}^{2 n+1}$ and the induced map $\bar{p}: M / G \rightarrow p(M)$ is a homeomorphism. Naturally, we set $Y=p(M)$, and we replace $M / G$ with $Y$. Next, we set $X=\operatorname{graph} p$ and let $\phi$ : $X \rightarrow Y$ denote the restriction to $X$ of the projection $M \times \mathbf{R}^{2 n+1} \rightarrow \mathbf{R}^{2 n+1}$. Then, as we have explained, $\phi$ is a subanalytic analytic map, and $X$ is a subanalytic $G$-set because the action is defined by $G \times M \times \mathbf{R}^{2 n+1} \supset G \times X \ni$ $(g, x, p(x)) \rightarrow(g x, p(x)) \in X \subset M \times \mathbf{R}^{2 n+1}$ and coincides with the restriction to $G \times X$ of the subanalytic analytic map $G \times M \times \mathbf{R}^{2 n+1} \ni(g, x, y) \rightarrow$ $(g x, y) \in M \times \mathbf{R}^{2 n+1}$. Thus, we can replace the map $\pi: M \rightarrow M / G$ with the one $\phi: X \rightarrow Y$.

We have modified the map $\pi: M \rightarrow M / G$ to the map $\phi: X \rightarrow Y$. We will show that the map $\phi$ satisfies the conditions in Theorem 2.1, apply Theorem 2.1, and obtain a subanalytic triangulation $(\tau: P \rightarrow X, \sigma: L \rightarrow$ $X / G)$ of $\phi$. Note that $X \subset \mathbf{R}^{2 n+1} \times \mathbf{R}^{2 n+1}$ and that $Y \subset \mathbf{R}^{2 n+1}$. First, we construct the canonical Whitney subanalytic analytic stratification of $\phi$ in the same way as in the case where condition $(*)$ is satisfied.

LEMMA 3.3. There exists the canonical Whitney subanalytic analytic stratification of $\phi$.

Proof. The most natural stratification of $\phi$ is the one by orbit types. Let $\left\{G_{i}\right\}_{i}$ be the family of compact subgroups of $G$, let $X_{i}$ be the subset of $X$ consisting of $x$ such that $G_{x}$ is conjugate to $G_{i}$ for each $i$, and set $Y_{i}=\phi\left(X_{i}\right)$. Then $X_{i}$ and $Y_{i}$ are subanalytic $C^{\omega}$ manifolds of various local dimension, and the set of indexes of $\left\{X_{i}\right\}_{i}$ is not necessarily finite, but by the fact in the proof of Lemma 3.1, the map $\phi:\left\{X_{i}\right\}_{i} \rightarrow\left\{Y_{i}\right\}_{i}$ satisfies the other conditions of a subanalytic analytic stratified map. We modify $\left\{X_{i}\right\}_{i}$ and $\left\{Y_{i}\right\}_{i}$ so that the last condition is satisfied. For each pair $(j, k)$ of nonnegative integers, let $Y_{j, k}$ denote the union of the connected components $C$ of all $Y_{i}$ such that $\operatorname{dim} C=j$ and $\operatorname{dim} \phi(C)=k$, and set $X_{j, k}=\phi^{-1}\left(Y_{j, k}\right)$. Then $\phi:\left\{X_{j, k}\right\}_{j, k} \rightarrow\left\{Y_{j, k}\right\}_{j, k}$ is the subanalytic analytic stratification of the map $\phi$ defined by orbit types. However, we do not know whether this stratified map satisfies the Whitney condition. Therefore, we define another stratification.

Set $X_{1}=\left.\operatorname{Reg} \phi\right|_{\operatorname{Reg} X}$, and set $Y_{1}=\phi\left(X_{1}\right)$. Then $X_{1}$ is a subanalytic analytic manifold of various local dimension, $\operatorname{dim}\left(X-X_{1}\right)<n$, and $X_{1}$ is $G$-invariant because (a) for each $(g, x) \in G \times X$ and for an open smooth 
neighborhood $U$ of $x$ in $X$, the map $U \ni y \rightarrow g y \in g U$ is an analytic diffeomorphism, (b) $g U$ is smooth, and (c) $\phi(x)=\phi\left(g^{-1} x\right)$ for $x \in g U$. Hence, $\phi^{-1}\left(Y_{1}\right)=X_{1}, Y_{1}$ is a subanalytic analytic manifold of various local dimension for the same reason and by the fact that the set $\phi(B)$ is subanalytic for each bounded subanalytic set $B \subset X \subset \mathbf{R}^{2 n+1} \times \mathbf{R}^{2 n+1}$, and $\left.\phi\right|_{X_{1}}$ is an analytic fiber bundle over each connected component of $Y_{1}$. It also follows that $\operatorname{dim}\left(Y-Y_{1}\right)<\operatorname{dim} Y$ because if $\operatorname{dim}\left(Y-Y_{1}\right)=\operatorname{dim} Y$, then there exists a subanalytic subset $U$ of $Y-Y_{1}$ which is open in $Y$, and hence $X_{1} \cap \phi^{-1}(U) \neq \emptyset$ and $X_{1}$ is not dense in $X$. For each $j, k=0, \ldots, n$, let $Y_{1, j, k}$ denote the union of the connected components $C$ of $Y_{1}$ of dimension $k$ such that $\operatorname{dim} \phi^{-1}(C)=j$. Set $X_{1, j, k}=\phi^{-1}\left(Y_{1, j, k}\right)$. Then $\left.\phi\right|_{X_{1}}:\left\{X_{1, j, k}\right\}_{j, k} \rightarrow$ $\left\{Y_{1, j, k}\right\}_{j, k}$ is a subanalytic analytic stratification of the map $\left.\phi\right|_{X_{1}}: X_{1} \rightarrow Y_{1}$. Moreover, since $X_{1, j, k}$ and $Y_{1, j, k}$ are the unions of some connected components of $X_{1}$ and $Y_{1}$, respectively, $X_{1, j, k} \cap \overline{X_{1, j^{\prime}, k^{\prime}}}=\emptyset$ and $Y_{1, j, k} \cap \overline{Y_{1, j^{\prime}, k^{\prime}}}=\emptyset$ for $(j, k) \neq\left(j^{\prime}, k^{\prime}\right)$, and hence $\left\{X_{1, j, k}\right\}_{j, k}$ and $\left\{Y_{1, j, k}\right\}_{j, k}$ satisfy the Whitney condition.

Apply the same arguments to the map $\left.\phi\right|_{X-X_{1}}: X-X_{1} \rightarrow Y-Y_{1}$. Then we have a subanalytic analytic manifold $X_{2}$ of various local dimension in $X-X_{1}$ and a subanalytic analytic stratification $\left.\phi\right|_{X_{2}}:\left\{X_{2, j, k}\right\}_{j, k} \rightarrow$ $\left\{Y_{2, j, k}\right\}_{j, k}$ of the map $\left.\phi\right|_{X_{2}}: X_{2} \rightarrow \phi\left(X_{2}\right)$ such that $\phi^{-1}\left(\phi\left(X_{2}\right)\right)=X_{2}$, $\operatorname{dim}\left(X-X_{1}-X_{2}\right)<\operatorname{dim}\left(X-X_{1}\right)$, and $\operatorname{dim}\left(Y-Y_{1}-\phi\left(X_{2}\right)\right)<\operatorname{dim}\left(Y-Y_{1}\right)$. Moreover, we require that $\left\{X_{1, j, k}, X_{2, j, k}\right\}_{j, k}$ and $\left\{Y_{1, j, k}, Y_{2, j, k}\right\}_{j, k}$ be Whitney stratifications. That is possible because for each $X_{2, j, k}$, the subsets of $X_{2, j, k}$ where some of $X_{1, j^{\prime}, k^{\prime}}$ and $X_{2, j, k}$ do not satisfy the Whitney condition and its closure in $X_{2, j, k}$ are $G$-invariant. In this way we obtain a Whitney subanalytic analytic stratification $\phi:\left\{X_{i, j, k}\right\}_{i, j, k} \rightarrow\left\{Y_{i, j, k}\right\}_{i, j, k}$ of the map $\phi: X \rightarrow Y$. It is easy to show that the stratification is canonical.

We simply write the canonical Whitney subanalytic analytic stratification as $\phi:\left\{X_{j}\right\}_{j} \rightarrow\left\{Y_{j}\right\}_{j}$. Note that points in one connected component of $X_{i}$ have the same orbit type. Next, we see the following.

Lemma 3.4. The stratified map $\phi:\left\{X_{j}\right\}_{j} \rightarrow\left\{Y_{j}\right\}_{j}$ is a Thom map.

Proof. Let $X_{j}$ and $X_{j^{\prime}}$ be strata such that $\overline{X_{j}} \cap X_{j^{\prime}} \neq \emptyset$, and let $\left\{a_{k}\right\}_{k}$ be a sequence of points in $X_{j}$ convergent to a point $b$ of $X_{j^{\prime}}$ such that $\left\{T_{a_{k}}\left(\left.\phi\right|_{X_{j}}\right)^{-1}\left(\phi\left(a_{k}\right)\right)\right\}_{k}$ converges to a space $T \subset \mathbf{R}^{2 n+1} \times \mathbf{R}^{2 n+1}$. Let each $a_{k}$ be described as $\left(a_{k}^{\prime}, p\left(a_{k}^{\prime}\right)\right) \in M \times \mathbf{R}^{2 n+1}$. Then $\left(\left.\phi\right|_{X_{j}}\right)^{-1}\left(\phi\left(a_{k}\right)\right)=$ $\phi^{-1}\left(\phi\left(a_{k}\right)\right)=G a_{k}$ since $X_{j}$ is $G$-invariant, and $G a_{k}=G a_{k}^{\prime} \times\left\{p\left(a_{k}^{\prime}\right)\right\}$. Hence, 
$T=\lim _{k \rightarrow \infty} T_{a_{k}^{\prime}} G a_{k}^{\prime} \times\{0\}$. Clearly $\lim _{k \rightarrow \infty} T_{a_{k}^{\prime}} G a_{k}^{\prime} \supset T_{b^{\prime}} G b^{\prime}$, where $b=\left(b^{\prime}\right.$, $\left.p\left(b^{\prime}\right)\right) \in M \times \mathbf{R}^{2 n+1}$. Therefore, $T_{b}\left(\left.\phi\right|_{X_{j^{\prime}}}\right)^{-1}(\phi(b)) \subset T$, and Lemma 3.4 is proved.

As we have noted, there always exists a strongly controlled subanalytic $C^{2+n}$ tube system $\left\{T_{Y, j}=\left(\left|T_{Y, j}\right|, \pi_{Y, j}, \rho_{Y, j}\right)\right\}_{j}$ for $\left\{Y_{j}\right\}_{j}$. From now on we fix one $\left\{T_{Y, j}\right\}_{j}$, although the domains $\left|T_{Y, j}\right|$ are shrunk many times, and naturally extend $\phi$ to the projection $\tilde{\phi}: \mathbf{R}^{2 n+1} \times \mathbf{R}^{2 n+1} \rightarrow \mathbf{R}^{2 n+1}$ to the latter factor. Then there is a subanalytic $C^{2+n}$ tube system $\left\{T_{X, j}=\right.$ $\left.\left(\left|T_{X, j}\right|, \pi_{X, j}, \rho_{X, j}\right)\right\}_{j}$ for $\left\{X_{j}\right\}_{j}$ strongly controlled over $\left\{T_{Y, j}\right\}_{j}$ since the map $\phi: X \rightarrow Y$ is analytic and the stratified map $\phi:\left\{X_{j}\right\}_{j} \rightarrow\left\{Y_{j}\right\}_{j}$ is a Thom map (see [14, Lemma I.1.3']). (It will be clarified why the class is $C^{2+n}$.) Hence, by Theorem 2.1, Theorem 1.1 holds if the following lemma does.

Lemma 3.5. We can choose a subanalytic $C^{2}$ tube system $\left\{T_{X, j}\right\}_{j}$ controlled over $\left\{T_{Y, j}\right\}_{j}$ so that the map $\left.\left(\pi_{X, j}, \phi\right)\right|_{X \cap\left|T_{X, j}\right|}: X \cap\left|T_{X, j}\right| \rightarrow X_{j} \times$ $\left|T_{Y, j}\right|$ is proper for each $j$, although $\left\{T_{X, j}\right\}_{j}$ becomes of class $C^{2}$.

In order to understand the problem of the construction of $\left\{T_{X, j}\right\}_{j}$, we consider the case of compact $G$.

Proof of Lemma 3.5 in the case of compact $G$. Remember that the map $\phi: X \rightarrow Y$ is proper. We know that there exists a subanalytic $C^{2+n}$ tube system $\left\{T_{X, j}=\left(\left|T_{X, j}\right|, \pi_{X, j}, \rho_{X, j}\right)\right\}_{j}$ for $\left\{X_{j}\right\}_{j}$ controlled over $\left\{T_{Y, j}\right\}_{j}$. We need to shrink $\left|T_{X, j}\right|$ and $\left|T_{Y, j}\right|$ so that the condition of properness in Lemma 3.5 is satisfied. First, we can assume that $\overline{\phi\left(X \cap\left|T_{X, j}\right|\right)} \subset\left|T_{Y, j}\right|$ and the set $\pi_{Y, j}^{-1}(y)$ are bounded for each $y \in Y_{j}$. For each $y \in Y_{j}$, set

$$
\chi_{j}(y)=\min \left\{\rho_{Y, j} \circ \phi(x)|x \in X \cap \partial| T_{X, j} \mid, \pi_{Y, j} \circ \phi(x)=y\right\},
$$

where $\partial\left|T_{X, j}\right|=\overline{\left|T_{X, j}\right|}-\left|T_{X, j}\right|$. Then $\chi_{j}$ is a function on $Y_{j}$ larger than some positive $C^{0}$ function since the map $\phi: X \rightarrow Y$ is proper, and its graph is subanalytic because the set $\left\{\left(x, \rho_{Y, j} \circ \phi(x), \pi_{Y, j} \circ(x)\right)|x \in X \cap \partial| T_{X, j} \mid\right\}$ is a subanalytic subset of $X \times \mathbf{R} \times Y$; hence, the set $\left\{\left(\rho_{Y, j} \circ \phi(x), \pi_{Y, j} \circ(x)\right) \mid\right.$ $\left.x \in X \cap \partial\left|T_{X, j}\right|\right\}$ is a subanalytic subset of $\mathbf{R} \times Y$ by (11) in Section 2, and in general, for a subanalytic subset $S$ of $\mathbf{R} \times Y$, the set $\{(y, s) \in Y \times \mathbf{R} \mid$ $s=\inf \{t \mid(t, y) \in S\}\}$ is subanalytic by (1). Therefore, by subanalytically stratifying the graph of $\chi_{j}$, we obtain a positive subanalytic function $\chi_{j}^{\prime}$ on $Y_{j}$ smaller than $\chi_{j}$. Let us shrink $\left|T_{Y, j}\right|$ and $\left|T_{X, j}\right|$ to the sets $\left\{y \in\left|T_{Y, j}\right| \mid\right.$ 
$\left.\rho_{Y, j}(y)<\chi_{j}^{\prime} \circ \pi_{Y, j}(y)\right\}$ and $\left|T_{X, j}\right| \cap\left(\mathbf{R}^{2 n+1} \times\left(\right.\right.$ the new $\left.\left.\left|T_{Y, j}\right|\right)\right)$, respectively. Then $\phi\left(X \cap\left|T_{X, j}\right|\right)=Y \cap\left|T_{Y, j}\right|$, and the set $X \cap\left|T_{X, j}\right|$ is $G$-invariant. Hence, the map $\left.\phi\right|_{X \cap\left|T_{X, j}\right|}: X \cap\left|T_{X, j}\right| \rightarrow\left|T_{Y, j}\right|$ is proper since the map $\phi: X \rightarrow Y$ is so. It follows that the map $\left.\left(\pi_{X, j}, \phi\right)\right|_{X \cap\left|T_{X, j}\right|}: X \cap\left|T_{X, j}\right| \rightarrow X_{j} \times\left|T_{Y, j}\right|$ is proper. Thus, Lemma 3.5 holds in the compact case.

The above arguments show that if $G$ is compact, then by shrinking $\left|T_{Y, j}\right|$ we can choose $\left|T_{X, j}\right|$ enough large in comparison. However, this is not the case in general (see Figure 7 ). In the general case, we will define $\left|T_{X, j}\right|$ only on slices, because isotropy groups are compact, and extend it globally. For the extension we need the additional condition that the restriction $\left.\pi_{X, j}\right|_{X \cap\left|T_{X, j}\right|}$ is $G$-equivariant, that is, that

$(* *)_{j} \quad g x \in\left|T_{X, j}\right|, \quad \pi_{X, j}(g x)=g \pi_{X, j}(x) \quad$ for $(g, x) \in G \times\left(X \cap\left|T_{X, j}\right|\right) ;$

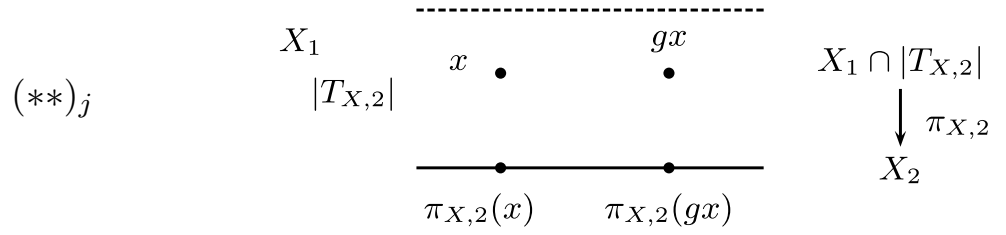

This condition is so strong that if the value $\pi_{X, j}(x)$ is determined for one $x$, then the map $\pi_{X, j}$ on the set $\phi^{-1}(\phi(x))$ is determined. Hence, if the condition is satisfied, then the map $\left.\left(\pi_{X, j}, \phi\right)\right|_{X \cap\left|T_{X, j}\right|}: X \cap\left|T_{X, j}\right| \rightarrow$ $X_{j} \times\left|T_{Y, j}\right|$ is proper. Thus, we can replace Lemma 3.5 with the following lemma.

Lemma 3.6. We can choose a subanalytic $C^{2}$ tube system $\left\{T_{X, j}\right\}_{j}$ controlled over $\left\{T_{Y, j}\right\}_{j}$ and satisfying condition $(* *)_{j}$.

We see that condition $(* *)_{j}$ can be satisfied in the case where $G$ is a compact subgroup of $\mathbf{O}(2 n+1)$ as follows, and we will reduce the general case to this case by existence of linear slices.

LEMMA 3.7. Assume that $G$ is a compact subgroup of the orthogonal group $\mathbf{O}(2 n+1)$ and that $G$ operates orthogonally on $M$. Then Lemma 3.6 holds.

Proof. Many times we use the idea of the construction of a controlled tube system by orthogonal projections onto differentiable submanifolds of $\mathbf{R}^{2 n+1}$. We construct $\left\{T_{X, j}\right\}_{j}$ by double induction. Choose the set of indexes 
so that $\operatorname{dim} X_{j} \geq \operatorname{dim} X_{j+1}$. Then, since we do not need to consider condition $(\mathrm{sc} 2)$, the required conditions are $(* *)_{j}$,

$$
\begin{aligned}
\phi \circ \pi_{X, j} & =\pi_{Y, j} \circ \tilde{\phi} \quad \text { on }\left|T_{X, j}\right|, \\
\pi_{X, j} \circ \pi_{X, k} & =\pi_{X, j} \quad \text { on }\left|T_{X, j}\right| \cap\left|T_{X, k}\right| \text { for } j>k .
\end{aligned}
$$

We also require that the map $\pi_{X, j}$ be of class $C^{*_{j}}$ for a technical reason, where $*_{j}=2+n-\operatorname{dim} X_{j}$.

As the first induction, we assume that for some $k$, a subanalytic tube system $\left\{T_{X, j}\right\}_{j>k}$ for $\left\{X_{j}\right\}_{j>k}$ is given so that the conditions $(\mathrm{sc} 1)_{j},(\mathrm{sc} 3)_{l, j}^{\prime}$, and $(* *)_{j}$ are satisfied and the map $\pi_{X, j}$ is of class $C^{*_{j}}$ for any $l>j>k$. We will define a subanalytic tube $T_{X, k}$ at $X_{k}$. First, we choose $\left|T_{X, k}\right|$ so small that $\left|T_{X, k}\right| \subset \tilde{\phi}^{-1}\left(\left|T_{Y, k}\right|\right)$; moreover, we shrink $\left|T_{X, k}\right|$ and, temporarily, define the value $\pi_{X, k}(x)$ for $x \in\left|T_{X, k}\right|$ to be the orthogonal projection of $x$ to the subanalytic analytic manifold $\phi^{-1}\left(\pi_{Y, k} \circ \tilde{\phi}(x)\right)$. Then $\pi_{X, k}$ is a submersive subanalytic $C^{1+n}$ retraction to $X_{k}$ by condition (14) because the family $\left\{\phi^{-1}\left(\pi_{Y, k} \circ \tilde{\phi}(x)\right)|x \in| T_{X, k} \mid\right\}$ is a subanalytic $C^{2+n}$ foliation of $X_{k}$ by condition (13); condition ( $\mathrm{sc} 1)_{k}$ is satisfied, and moreover, $(* *)_{k}$ is satisfied since $G$ acts orthogonally on $M \times \mathbf{R}^{2 n+1}$. However, condition $(\mathrm{sc} 3)_{j, k}^{\prime}$ is not necessarily satisfied for $j>k$. As the second induction, we assume that for some $l>k$ we have shrunk $\left|T_{X, j}\right|, l>j>k$, and have modified the map $\pi_{X, k}$ so that condition $(\mathrm{sc} 3)_{j, k}^{\prime}(l>j>k)$ is also satisfied and the map $\pi_{X, k}$ is now of class subanalytic $C^{*_{k}}$. Then we will modify $\pi_{X, k}$ again so that condition (sc3) $)_{l, k}^{\prime}$ is also satisfied. (In the accompanying arguments we need to shrink $\left|T_{X, j}\right|, l \geq j \geq k$, many times. However, we do not mention that anymore because it is clear when we need to do so.)

We can assume that $\operatorname{dim} X_{k}>\operatorname{dim} X_{l}$ because if $\operatorname{dim} X_{k}=\operatorname{dim} X_{l}$, then we can choose disjoint $\left|T_{X, k}\right|$ and $\left|T_{X, l}\right|$. For each $x \in\left|T_{X, k}\right| \cap\left|T_{X, l}\right|$, the set

$$
X_{k, l, x}=\pi_{X, l}^{-1}\left(\pi_{X, l}(x)\right) \cap \phi^{-1}\left(\pi_{Y, k} \circ \tilde{\phi}(x)\right)
$$

is a subanalytic $C^{*_{l}}$ submanifold of $X_{k} \cap\left|T_{X, l}\right|$ since $\phi$ is a Thom map; the family $\left\{X_{k, l, x}|x \in| T_{X, k}|\cap| T_{X, l} \mid\right\}$ is a subanalytic $C^{*_{l}}$ foliation of $X_{k} \cap\left|T_{X, l}\right|$ by (13); and any point $x$ in $X_{k} \cap\left|T_{X, l}\right|$ is contained in $X_{k, l, x}$. Let $p_{k, l, x}$ denote the orthogonal projection to $X_{k, l, x}$ of its small subanalytic neighborhood, and set

$$
\pi_{X, k, l}(x)=p_{k, l, x}\left(\pi_{X, k}(x)\right) \quad \text { for } x \in\left|T_{X, k}\right| \cap\left|T_{X, l}\right| .
$$

Then the map $\pi_{X, k, l}:\left|T_{X, k}\right| \cap\left|T_{X, l}\right| \rightarrow X_{k} \cap\left|T_{X, l}\right|$ is a well-defined subanalytic submersive $C^{*_{l}-1}$ retraction by condition (14); the conditions $(\mathrm{sc} 1)_{k}$ 
for $\pi_{X, k, l}$ and $(\mathrm{sc} 3)_{l, k}^{\prime}$ for $\pi_{X, l}$ and $\pi_{X, k, l}$ are clear; condition $(* *)_{k}$ for $\pi_{X, k, l}$ holds because for $(g, x) \in G \times\left(X \cap\left|T_{X, k}\right| \cap\left|T_{X, l}\right|\right)$, the following holds:

$$
\begin{aligned}
\pi_{X, k, l}(g x) & =p_{k, l, g x}\left(\pi_{X, k}(g x)\right)=p_{k, l, g x}\left(g \pi_{X, k}(x)\right) \quad \text { by }(* *)_{k} \text { for } \pi_{X, k} \\
& =g p_{k, l, x}\left(\pi_{X, k}(x)\right)
\end{aligned}
$$

since $p_{k, l, g x}\left(g x^{\prime}\right)=g p_{k, l, x}\left(x^{\prime}\right)$ for $x^{\prime}$ near $X_{k, l, x}$.

However, there are two problems when we choose the map $\pi_{X, k, l}$ as a modification of $\pi_{X, k}$. One is that condition $(\mathrm{sc} 3)_{j, k}^{\prime}, l>j>k$, may not continue to hold, and the other is that the map $\pi_{X, k, l}$ is not globally defined on $\left|T_{X, k}\right|$.

The former problem does not occur. Indeed, for any $j$ such that $l>j>k$, we have the equality

$(* * *) \quad \pi_{X, k, l}=\pi_{X, k} \quad$ on $\left|T_{X, k}\right| \cap\left|T_{X, l}\right| \cap\left|T_{X, j}\right|$

as follows. Let $x \in\left|T_{X, k}\right| \cap\left|T_{X, l}\right| \cap\left|T_{X, j}\right|$. Then

$$
\begin{aligned}
& \pi_{X, l}(x) \stackrel{\text { by }}{(\mathrm{sc} 3)_{l, j}^{\prime}} \pi_{X, l} \circ \pi_{X, j}(x) \stackrel{\text { by }}{=} \stackrel{(\mathrm{sc} 3)_{j, k}^{\prime}}{=} \pi_{X, l} \circ \pi_{X, j} \circ \pi_{X, k}(x)
\end{aligned}
$$

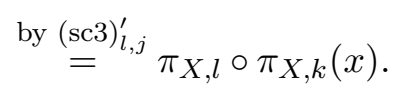

Hence, $\pi_{X, k}(x) \in \pi_{X, l}^{-1}\left(\pi_{X, l}(x)\right)$. On the other hand, $\pi_{X, k}(x) \in \phi^{-1}\left(\pi_{Y, k} \circ\right.$ $\tilde{\phi}(x))$ by condition $(\mathrm{sc} 1)_{k}$. Consequently, $\pi_{X, k}(x) \in X_{k, l, x}$, which proves the equality $(* * *)$ by the definition of $\pi_{X, k, l}$.

To solve the latter problem, we paste two maps $\pi_{X, k}$ and $\pi_{X, k, l}$ by a partition of unity. We use a special partition of unity so that conditions $(\mathrm{sc} 1)_{k}$ and $(* *)_{k}$ continue to hold. Let $\xi$ be a subanalytic $C^{2+n}$ function defined on $\left|T_{Y, l}\right|$ (not $\left|T_{X, l}\right|$ ) such that $\xi \geq 0, \xi=0$ outside of a small subanalytic neighborhood of $Y_{l}$ in $\tilde{\phi}\left(\left|T_{X, l}\right|\right)$ and $\xi=1$ on a smaller one. It is natural to define the pasted map to be $\pi_{X, k}$ on $\left|T_{X, k}\right|-\left|T_{X, l}\right|$ and $\xi \circ \tilde{\phi} \pi_{X, k, l}+(1-\xi \circ \tilde{\phi}) \pi_{X, k}$ on $\left|T_{X, k}\right| \cap\left|T_{X, l}\right|$. However, this map loses the required properties. We need to modify the map. Set

$$
\pi_{X, k}^{\prime}(x)= \begin{cases}\pi_{X, k}(x) & \text { for } x \in\left|T_{X, k}\right|-\left|T_{X, l}\right|, \\ p_{k, x}\left(\xi \circ \tilde{\phi}(x) \pi_{X, k, l}(x)\right. & \\ \left.\quad+(1-\xi \circ \tilde{\phi}(x)) \pi_{X, k}(x)\right) & \text { for } x \in\left|T_{X, k}\right| \cap\left|T_{X, l}\right|\end{cases}
$$


where $p_{k, x}$ denotes the orthogonal projection to the manifold $\phi^{-1}\left(\pi_{Y, k} \circ\right.$ $\tilde{\phi}(x))$ of its small subanalytic neighborhood. Then $\pi_{X, k}^{\prime}$ is the required modification of $\pi_{X, k}$. Indeed, the map $\pi_{X, k}^{\prime}:\left|T_{X, k}\right| \rightarrow X_{k}$ is a subanalytic submersive $C^{*_{k}}$ retraction; $\pi_{X, k}^{\prime}$ clearly satisfies conditions $(\mathrm{sc} 1)_{k}$ and $(\mathrm{sc} 3)_{l, k}^{\prime}$; and $\pi_{X, k}^{\prime}=\pi_{X, k}$ on $\left|T_{X, k}\right| \cap\left|T_{X, j}\right|$ for $l>j>k$ by $(* * *)$. Hence, condition $(\operatorname{sc} 3)_{j, k}^{\prime}$ holds for $l>j>k$ by the same reason as above, and condition $(* *)_{k}$ for $\pi_{X, k}^{\prime}$ holds for the same reason as above and the fact that $\xi \circ \tilde{\phi}=$ const on $G x$ for each $x \in X$. Thus, the second induction works, there exists a subanalytic $C^{2}$ tube system $\left\{T_{X, j}\right\}_{j}$ controlled over $\left\{T_{Y, j}\right\}_{j}$ and satisfying $(* *)_{j}$, and Lemma 3.7 is proved.

We simplify Lemma 3.6 as follows.

LEMma $3.6^{\prime}$. There exist subanalytic $C^{2}$ submersive retractions $\pi_{X, j}$ : $\left|T_{X, j}\right| \cap\left(M \times \mathbf{R}^{2 n+1}\right) \rightarrow X_{j}$ such that

$$
\begin{aligned}
\phi \circ \pi_{X, j} & =\pi_{Y, j} \circ \tilde{\phi} \quad \text { on }\left|T_{X, j}\right| \cap\left(M \times \mathbf{R}^{2 n+1}\right), \\
\pi_{X, j} \circ \pi_{X, j^{\prime}} & =\pi_{X, j} \quad \text { on }\left|T_{X, j}\right| \cap\left|T_{X, j^{\prime}}\right| \cap\left(M \times \mathbf{R}^{2 n+1}\right),
\end{aligned}
$$

and condition $(* *)_{j}$ is satisfied, where $\left|T_{X, j}\right|$ are subanalytic tubular neighborhoods of $X_{j}$ in $\mathbf{R}^{2 n+1} \times \mathbf{R}^{2 n+1}$.

Proof that Lemma 3.6' implies Lemma 3.6. Assume that we have constructed $\pi_{X, j}$ as required in Lemma $3.6^{\prime}$. Define an extension $\tilde{\pi}_{X, j}$ of $\pi_{X, j}$ to be $\pi_{X, j} \circ(q$,id $)$ on $\left|T_{X, j}\right| \cap\left(U \times \mathbf{R}^{2 n+1}\right)$, where $q: U \rightarrow M$ is the orthogonal projection of a subanalytic tubular neighborhood $U$ of $M$ in $\mathbf{R}^{2 n+1}$. Then the following hold on $\left|T_{X, j}\right| \cap\left(U \times \mathbf{R}^{2 n+1}\right)$ :

$$
\begin{aligned}
\phi \circ \tilde{\pi}_{X, j} & =\phi \circ \pi_{X, j} \circ(q, \mathrm{id})=\pi_{Y, j} \circ \tilde{\phi} \circ(q, \mathrm{id})=\pi_{Y, j} \circ \tilde{\phi}, \\
\tilde{\pi}_{X, j} \circ \tilde{\pi}_{X, j^{\prime}} & =\pi_{X, j} \circ(q, \mathrm{id}) \circ \pi_{X, j^{\prime}} \circ(q, \mathrm{id}) \\
& =\pi_{X, j} \circ \pi_{X, j^{\prime}} \circ(q, \mathrm{id})=\pi_{X, j} \circ(q, \mathrm{id})=\tilde{\pi}_{X, j} ;
\end{aligned}
$$

and condition $(* *)_{j}$ continues to hold. Hence, $\left\{\tilde{\pi}_{X, j}\right\}_{j}$ fulfills the requirements in Lemma 3.6.

Proof of Lemma 3. $6^{\prime}$ in the case of noncompact $G$. For each point $a \in M$, there exists a linear slice $S$ at $a$ (see [1, Chapter IV] and [12, Theorem 2.3.3]). Here we can clearly choose $S$ bounded and subanalytic. Let $a_{\alpha}(\alpha \in A)$ be a finite or countable number of points of $M$ and $S_{\alpha}$ bounded subanalytic lin- 
ear slices at $a_{\alpha}$ such that $\left\{p\left(S_{\alpha}\right)\right\}_{\alpha \in A}$ is a locally finite covering of $Y$, where $p: M \rightarrow \mathbf{R}^{2 n+1}$ is the map which appeared in the proof of Lemma 3.2.

The above construction of the stratified map $\phi:\left\{X_{j}\right\}_{j} \rightarrow\left\{Y_{j}\right\}_{j}$ works under the additional condition of compatibility. Hence, we can assume that the stratified map $\phi:\left\{X_{j}\right\}_{j} \rightarrow\left\{Y_{j}\right\}_{j}$ is compatible with $\left\{X \cap\left(G S_{\alpha} \times \mathbf{R}^{2 n+1}\right)\right\}_{\alpha}$ and $\left\{p\left(S_{\alpha}\right)\right\}_{\alpha}$, that is, that each of $X \cap\left(G S_{\alpha} \times \mathbf{R}^{2 n+1}\right)$ or $p\left(S_{\alpha}\right)$ is the union of some connected components of $X_{j}$ or $Y_{j}$, respectively. Moreover, we suppose that each of $X \cap\left(G S_{\alpha} \times \mathbf{R}^{2 n+1}\right)$ or $p\left(S_{\alpha}\right)$ is the union of some of $X_{j}$ or $Y_{j}$, respectively, and postpone considering the general case. For each $j$, let $\alpha_{j}$ be an element of $A$ such that $X_{j} \subset G S_{\alpha_{j}} \times \mathbf{R}^{2 n+1}$. Here we can assume, moreover, that $\overline{X_{j}} \subset G S_{\alpha_{j}} \times \mathbf{R}^{2 n+1}$ without loss of generality. Set $a_{j}=a_{\alpha_{j}}, S_{j}=S_{\alpha_{j}}, G_{j}=G_{a_{j}}$, and $Z_{j}=\left.\operatorname{graph} p\right|_{S_{j}}$ for each $j$. Note that $X_{j} \neq X_{j^{\prime}}$ for $j \neq j^{\prime}$, but there is the case where $a_{j}=a_{j^{\prime}}, S_{j}=S_{j^{\prime}}$, and $G_{j}=G_{j^{\prime}}$ for $j \neq j^{\prime}$, and the compact Lie group $G_{j}$ and the $G_{j^{-m a n i f o l d}} S_{j}$ satisfy the conditions in Lemma 3.7 for each $j$. Choose the set of indexes so that $\operatorname{dim} X_{j} \geq \operatorname{dim} X_{j+1}$ as usual. We will construct a subanalytic tube system $\left\{T_{X, j}\right\}_{j}$ by double induction as in the proof of Lemma 3.7. Assume that for some $k$ we are given $T_{X, j}$ on $\left|T_{X, j}\right| \cap\left(M \times \mathbf{R}^{2 n+1}\right), j>k$, of class $C^{*_{j}}$ such that the conditions $(\mathrm{sc} 1)_{j},(\mathrm{sc} 3)_{l, j}^{\prime}$, and $(* *)_{j}$ are satisfied for any $l>j>k$. We will define $T_{X, k}$.

Here, too, we do not mention shrinking $\left|T_{X, j}\right|$ and $\left|T_{Y, j}\right|$ each time, though we need to keep the condition $\phi^{-1}\left(\left|T_{Y, j}\right|\right)=X \cap\left|T_{X, j}\right|$. First, define a subanalytic $C^{1+n}$ tube $T_{Z, k}=\left(\left|T_{Z, k}\right|, \pi_{Z, k}, \rho_{Z, k}\right)$ at $Z_{k} \cap X_{k}$ in $S_{k} \times \mathbf{R}^{2 n+1}$ so that the value $\pi_{Z, k}(z)$ is the orthogonal projection of a point $z$ to the analytic manifold $Z_{k} \cap \phi^{-1}\left(\pi_{Y, k} \circ \phi(z)\right)$, where $S_{k}$ is regarded as a Euclidean space and $G_{k}$ acts orthogonally there and trivially on $\mathbf{R}^{2 n+1}$. As in the case of compact $G$, choose $\left|T_{Y, k}\right|$ and $\left|T_{Z, k}\right|$ so that $Z_{k} \cap \phi^{-1}\left(\left|T_{Y, k}\right|\right)=Z_{k} \cap\left|T_{X, k}\right|$, which is possible because $\left\{g \in G \mid g Z_{k}=Z_{k}\right\}=G_{k}$ and $G_{k}$ is compact. Then condition $(\mathrm{sc} 1)_{k}$ for $\pi_{Z, k}$ is satisfied, and we have

$(* *)_{Z, k}$

$$
\begin{aligned}
& g z \in Z_{k} \cap\left|T_{Z, k}\right| \quad \text { and } \quad \pi_{Z, k}(g z)=g \pi_{Z, k}(z) \\
& \quad \text { for }(g, z) \in G_{k} \times\left(Z_{k} \cap\left|T_{Z, k}\right|\right)
\end{aligned}
$$

since $G_{k}$ acts orthogonally on $Z_{k}$.

Next, we extend $T_{Z, k}$ to a subanalytic $C^{1+n}$ tube $T_{X, k}$ at $X_{k}$. For that, it suffices to define the map $\pi_{X, k}$ on $\left|T_{X, k}\right| \cap\left(M \times \mathbf{R}^{2 n+1}\right)$. Choose $\left|T_{X, k}\right|$ so that

$$
\left|T_{X, k}\right| \cap\left(M \times \mathbf{R}^{2 n+1}\right)=G\left|T_{Z, k}\right|,
$$


and set

$$
\pi_{X, k}(g z)=g \pi_{Z, k}(z) \quad \text { for }(g, z) \in G \times\left|T_{Z, k}\right| .
$$

First of all, the map $\pi_{X, k}$ is then well defined. Indeed, if $g z=g^{\prime} z^{\prime}$ for points $(g, z),\left(g^{\prime}, z^{\prime}\right) \in G \times\left|T_{Z, k}\right|$, then points $s$ and $s^{\prime}$ in $S_{k}$ such that $z=(s, p(s))$ and $z^{\prime}=\left(s^{\prime}, p\left(s^{\prime}\right)\right)$ satisfy the equation $s=g^{-1} g^{\prime} s^{\prime}$; hence, $g^{-1} g^{\prime} \in G_{k}$ since $G \times{ }_{G_{k}} S_{k} \rightarrow M$ is an embedding. Therefore,

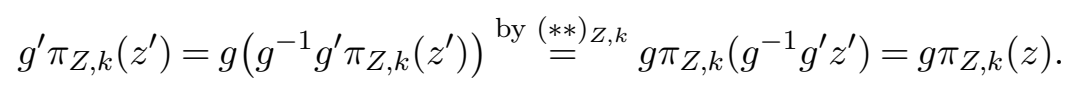

Second, the map $\pi_{X, k}:\left|T_{X, k}\right| \cap\left(M \times \mathbf{R}^{2 n+1}\right) \rightarrow X_{k}$ is clearly a subanalytic submersive $C^{1+n}$ retraction. Finally,

$$
\begin{aligned}
G \operatorname{Dom} \pi_{X, k} & =\operatorname{Dom} \pi_{X, k}, \pi_{X, k}\left(g g^{\prime} z\right)=g g^{\prime} \pi_{Z, k}(z) \\
& =g \pi_{X, k}\left(g^{\prime} z\right) \quad \text { for }\left(g, g^{\prime}, z\right) \in G^{2} \times\left|T_{Z, k}\right| .
\end{aligned}
$$$$
\phi \circ \pi_{X, k}(g z)=\phi\left(g \pi_{Z, k}(z)\right)=\phi \circ \pi_{Z, k}(z)
$$$$
(\mathrm{sc} 1)_{k} \quad \text { by }(\mathrm{sc} 1)_{k} \text { for } \pi_{Z, k} \pi_{Y, k} \circ \phi(z)=\pi_{Y, k} \circ \phi(g z)
$$$$
\text { for }(g, z) \in G \times\left|T_{Z, k}\right|,
$$

Thus, we extend $T_{Z, k}$ to $T_{X, k}$.

We need to modify $\pi_{X, k}$ so that condition $(\operatorname{sc} 3)_{j, k}^{\prime}$ for $\pi_{X, j}$ and $\pi_{X, k}$ holds for any $j>k$. By downward induction we assume that for some $l>k$ and for any $j$ such that $l>j>k$, condition $(\operatorname{sc} 3)_{j, k}^{\prime}$ is satisfied and the map $\pi_{X, k}$ is of class $C^{*_{k}}$. We modify $\pi_{X, k}$ so that condition $(\mathrm{sc} 3)_{l, k}^{\prime}$ is also satisfied. However, we cannot carry out the modification in $Z_{k}$ as in the proof of Lemma 3.7 because $S_{l}$ and $S_{k}$ may be different, although we assume that $X_{l} \subset \overline{X_{k}}-X_{k}$ without loss of generality. We will replace $\pi_{Z, k}$ with some subanalytic $C^{*_{k}}$ map $\pi_{Z, k}^{\prime}: Z_{k} \cap\left|T_{X, l}\right| \rightarrow X_{k}$ (not a map to $Z_{k} \cap X_{k}$ ) and extend it to a subanalytic $C^{*_{k}} \operatorname{map} \pi_{X, k}^{\prime}:\left|T_{X, k}\right| \cap\left(M \times \mathbf{R}^{2 n+1}\right) \rightarrow X_{k}$ in the same way as above so that condition $(\operatorname{sc} 3)_{l, k}^{\prime}$ is satisfied for $\pi_{X, l}$ and $\pi_{X, k}^{\prime}$.

In the proof of Lemma 3.7 we constructed $\pi_{X, k, l}$ before $\pi_{X, k}^{\prime}$. Here we construct a map $\pi_{Z, k, l}$ having the same properties as $\pi_{X, k, l}$. Set $\pi_{Z, k}=$ $\left.\pi_{X, k}\right|_{Z_{k} \cap\left|T_{X, k}\right|}$. (Note that the present $\pi_{Z, k}$ is not the original $\pi_{Z, k}$ but the restriction to $Z_{k} \cap\left|T_{X, k}\right|$ of the modified $\pi_{X, k}$.) We need a revision of the orthogonal direction to $S_{k}$ in $M$. Regard $M$ as a subanalytic analytic $G_{k^{-}}$ manifold, give it an analytic $G_{k}$-invariant Riemannian metric (see [1, Chapter VI, proof of Theorem 2.1]), and trivially lift the metric to $M \times \mathbf{R}^{2 n+1}$. 
Since $Z_{k}$ is bounded, for each $z \in Z_{k} \cap\left|T_{X, k}\right| \cap\left|T_{X, l}\right|$, the set

$$
\tilde{Z}_{k, l, z}=\pi_{X, l}^{-1}\left(\pi_{X, l}(z)\right) \cap \phi^{-1}\left(\pi_{Y, k} \circ \phi(z)\right)
$$

is a subanalytic $C^{*_{l}}$ submanifold of $X_{k} \cap\left|T_{X, l}\right|$, any point $z$ in $Z_{k} \cap X_{k} \cap$ $\left|T_{X, l}\right|$ is included in $\tilde{Z}_{k, l, z}$, and the family $\left\{\tilde{Z}_{k, l, z}\left|z \in Z_{k} \cap X_{k} \cap\right| T_{X, l} \mid\right\}$ is a subanalytic $C^{*_{l}}$ foliation of $X_{k} \cap\left|T_{X, l}\right|$. Let $p_{k, l, z}$ denote the projection to $\tilde{Z}_{k, l, z}$ of its small subanalytic neighborhood such that for each $z^{\prime} \in \tilde{Z}_{k, l, z}$, $p_{k, l, z}^{-1}\left(z^{\prime}\right)$ is a geodesic curve in the neighborhood and orthogonal to $\tilde{Z}_{k, l, z}$ at $z^{\prime}$, and set

$$
\pi_{Z, k, l}(z)=p_{k, l, z}\left(\pi_{Z, k}(z)\right) \quad \text { for } z \in Z_{k} \cap\left|T_{X, k}\right| \cap\left|T_{X, l}\right| \text {. }
$$

Then $\pi_{Z, k, l}: Z_{k} \cap\left|T_{X, k}\right| \cap\left|T_{X, l}\right| \rightarrow X_{k}$ is a well-defined subanalytic $C^{*_{l}}$ map such that $\pi_{Z, k, l}=$ id on $Z_{k} \cap X_{k} \cap\left|T_{X, l}\right|$; the conditions $(\operatorname{sc} 1)_{k}$ for $\pi_{X, k, l}$ and $(\mathrm{sc} 3)_{l, k}^{\prime}$ for $\pi_{X, l}$ and $\pi_{Z, k, l}$ on $Z_{k} \cap\left|T_{X, k}\right| \cap\left|T_{X, l}\right|$ are clearly satisfied; and condition $(* *)_{Z, k}$ holds on $G_{k} \times\left(Z_{k} \cap\left|T_{X, k}\right| \cap\left|T_{X, l}\right|\right)$ for the same reason as in the proof of Lemma 3.7. The following equality also follows in the same way. For each $j$ such that $l>j>k$,

$$
\pi_{Z, k, l}=\pi_{Z, k} \quad \text { on } Z_{k} \cap\left|T_{X, l}\right| \cap\left|T_{X, j}\right| \cap\left|T_{X, k}\right| .
$$

Thus, the map $\pi_{Z, k, l}$ on $Z_{k} \cap\left|T_{X, k}\right| \cap\left|T_{X, l}\right|$ has the same properties as $\pi_{X, k, l}$. (Note that $\operatorname{Im} \pi_{Z, k, l}$ is not necessarily equal to $Z_{k} \cap X_{k}$ and that the map $\pi_{Z, k, l}$ is not necessarily a submersion to its image.)

It remains to paste two maps $\pi_{Z, k, l}$ with $\pi_{Z, k}$. As before, let $\xi$ be a subanalytic $C^{2+n}$ function defined on $\left|T_{Y, l}\right|$ such that $\xi \geq 0, \xi=0$ outside of a small subanalytic neighborhood of $Y_{l}$ in $\left|T_{Y, l}\right|$ and $\xi=1$ on a smaller one. Let $\left(x, x^{\prime}, t\right)$ be a point of $\left(M \times \mathbf{R}^{2 n+1}\right)^{2} \times[0,1]$ such that $x$ and $x^{\prime}$ are close each other. Let $\theta\left(x, x^{\prime}, t\right) \in M \times \mathbf{R}^{2 n+1}$ denote the point in the shortest geodesic curve joining $x$ and $x^{\prime}$ such that the distance between $x$ and $\theta\left(x, x^{\prime}, t\right)$ equals the product of $t$ and the distance between $x$ and $x^{\prime}$. Set

$$
\pi_{Z, k}^{\prime}(z)= \begin{cases}\pi_{Z, k}(z) & \text { for } z \in Z_{k} \cap\left|T_{X, k}\right|-\left|T_{X, l}\right|, \\ p_{k, z} \circ \theta\left(\pi_{Z, k}(z), \pi_{Z, k, l}(z), \xi \circ \phi(z)\right) & \text { for } z \in Z_{k} \cap\left|T_{X, k}\right| \cap\left|T_{X, l}\right|,\end{cases}
$$

where $p_{k, z}$ denotes the projection to the manifold $\phi^{-1}\left(\pi_{Y, k} \circ \phi(z)\right)$ of its small subanalytic neighborhood with the same properties as $p_{k, l, z}$. Then the $\operatorname{map} \pi_{Z, k}^{\prime}: Z_{k} \cap\left|T_{X, k}\right| \rightarrow X_{k}$ is a subanalytic $C^{*_{k}}$ one, and all the conditions 
$\pi_{Z, k}^{\prime}=$ id on $Z_{k} \cap X_{k},(\operatorname{sc} 1)_{k}$ for $\pi_{Z, k}^{\prime}$ on $Z_{k} \cap\left|T_{X, k}\right|,(\operatorname{sc} 3)_{j, k}^{\prime}$ for $\pi_{X, j}$ and $\pi_{Z, k}^{\prime}(l \geq j>k)$ on $Z_{k} \cap\left|T_{X, j}\right| \cap\left|T_{X, k}\right|$, and $(* *)_{Z, k}$ for $\pi_{Z, k}^{\prime}$ hold.

Extend $\pi_{Z, k}^{\prime}$ to a subanalytic $C^{*_{k}}$ map $\pi_{X, k}^{\prime}:\left|T_{X, k}\right| \cap\left(M \times \mathbf{R}^{2 n+1}\right) \rightarrow X_{k}$ in the same way as before by

$$
\pi_{X, k}^{\prime}(g z)=g \pi_{Z, k}^{\prime}(z) \quad \text { for }(g, z) \in G \times\left(Z_{k} \cap\left|T_{X, k}\right|\right) .
$$

Then for the same reason, the map $\pi_{X, k}^{\prime}$ is a well-defined submersive retraction; the conditions $(\mathrm{sc} 1)_{k}$ and $(* *)_{X, k}$ are satisfied; condition $(\mathrm{sc} 3)_{j, k}^{\prime}$ for $\pi_{X, j}$ and $\pi_{X, k}^{\prime}(l>j>k)$ holds because

$$
\pi_{X, k}^{\prime}=\pi_{X, k} \quad \text { on }\left|T_{X, k}\right| \cap\left|T_{X, j}\right| \cap\left(M \times \mathbf{R}^{2 n+1}\right) ;
$$

and finally,

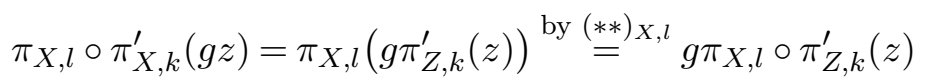

$$
\begin{aligned}
& (\mathrm{sc} 3)_{l, k}^{\prime} \quad \text { by }(\mathrm{sc} 3)_{l, k}^{\prime} \stackrel{\text { for } \pi_{X, l}}{=} \text { and } \pi_{Z, k}^{\prime} g \pi_{X, l}(z)
\end{aligned}
$$

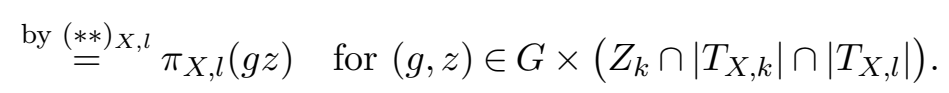

Thus, $\pi_{X, k}^{\prime}$ is the required modification of $\pi_{X, k}$ in the induction process, completing the proof of Lemma 3.6' in the case where each of $X \cap\left(G S_{\alpha} \times\right.$ $\left.\mathbf{R}^{2 n+1}\right)$ or $p\left(S_{\alpha}\right)$ is the union of some $X_{j}$ or $Y_{j}$, respectively.

Let us consider the general case where each is the union of some connected components of $X_{j}$ or $Y_{j}$. For each $j$, let $\left\{Y_{i, j}\right\}_{i}$ denote the connected components of $Y_{j}$, and set $X_{i, j}=\phi^{-1}\left(Y_{i, j}\right)$. Then by condition (6), the families $\left\{X_{i, j}\right\}_{i, j}$ and $\left\{Y_{i, j}\right\}_{i, j}$ satisfy the conditions on a subanalytic analytic stratification except that the set of indexes is finite. By (5) the families are locally finite, and the map $\phi:\left\{X_{i, j}\right\}_{i, j} \rightarrow\left\{Y_{i, j}\right\}_{i, j}$ satisfies the condition on a subanalytic analytic stratified map. For each $(i, j)$, since $Y_{i, j}$ is a connected component of $Y_{j}$, we can define a tube $T_{Y, i, j}=\left(\left|T_{Y, i, j}\right|, \pi_{Y, i, j}, \rho_{Y, i, j}\right)$ at $Y_{i, j}$ to be the restriction of $T_{Y, j}$ to the neighborhood of $Y_{i, j}$; that is, $\left|T_{Y, i, j}\right|=\pi_{Y, j}^{-1}\left(Y_{i, j}\right), \pi_{Y, i, j}=\left.\pi_{Y, j}\right|_{\left|T_{Y, i, j}\right|}$ and $\rho_{Y, i, j}=\left.\rho_{Y, j}\right|_{\left|T_{Y, i, j}\right|}$. Then $\left\{T_{Y, i, j}\right\}_{i, j}$ is a controlled subanalytic $C^{2+n}$ tube system for $\left\{Y_{i, j}\right\}_{i, j}$. Hence, it suffices to find a subanalytic $C^{2}$ tube system $\left\{T_{X, i, j}\right\}_{i, j}$ for $\left\{X_{i, j}\right\}_{i, j}$ controlled over $\left\{T_{Y, i, j}\right\}_{i, j}$ and satisfying condition $(* *)$.

By induction, we assume that the required $\left\{T_{X, i, j} \mid \operatorname{dim} X_{i, j}<k\right\}$ is given for some $k$, and we construct $\left\{T_{X, i, j} \mid \operatorname{dim} X_{i, j}=k\right\}$. Since the manifold 
$\bigcup_{\operatorname{dim} X_{i, j}=k} X_{i, j}$ is not necessarily included in one $G S_{\alpha} \times \mathbf{R}^{2 n+1}$, we need to choose one $\alpha \in A$, say, $\alpha_{i, j}$ for each $(i, j)$ such that $X_{i, j}$ is included in $G S_{\alpha_{i, j}} \times \mathbf{R}^{2 n+1}$. Then by using $S_{\alpha_{i, j}}$ and $G_{\alpha_{i, j}}$ for each $(i, j)$ such that $\operatorname{dim} X_{i, j}=k$, we obtain the required $T_{X_{i, j}}$ in the same way as in the special case, though the notation becomes complicated. We omit the details.

\section{REFERENCES}

[1] G. E. Bredon, Introduction to Compact Transformation Groups, Pure Appl. Math. 46. Academic Press, New York, 1972. MR 0413144.

[2] C. G. Gibson, K. Wirthmüller, A. A. du Plessis, and E. J. N. Looijenga, Topological Stability of Smooth Mappings, Lecture Notes in Math. 552, Springer, Berlin, 1976. MR 0436203.

[3] H. Grauert, On Levi's problem and the imbedding of real-analytic manifolds, Ann. of Math. (2) 68 (1958), 460-472. MR 0098847.

[4] H. Hironaka, "Subanalytic sets" in Number Theory, Algebraic Geometry and Commutative Algebra, in Honor of Yasuo Akizuki, Kinokuniya, Tokyo, 1973, 453-493. MR 0377101.

[5] S. Illman, "Every proper smooth action of a Lie group is equivalent to a real analytic action" in Prospects in Topology (Princeton, 1994), Ann. of Math. Stud. 138, Princeton University Press, Princeton, 1995, 189-220. MR 1368660.

[6] J. L. Koszul, "Sur certains groupes de transformations de Lie" in Géométrie différentielle (Strasbourg, 1953), Centre National de la Recherche Scientifique, Paris, 1953, 137-141. MR 0059919.

[7] S. Łojasiewicz, Triangulation of semi-analytic sets, Ann. Sc. Norm. Super. Pisa Cl. Sci. (3) 18 (1964), 449-474. MR 0173265.

[8] T. Matumoto and M. Shiota, "Proper subanalytic transformation groups and unique triangulation of the orbit spaces" in Transformation Groups (Poznań, 1985), Lecture Notes in Math. 1217, Springer, Berlin, 1986, 290-302. MR 0874184. DOI 10.1007/ BFb0072829.

[9] - "Unique triangulation of the orbit space of a differentiable transformation group and its applications" in Homotopy Theory and Related Topics (Kyoto, 1984), Adv. Stud. Pure Math. 9, North-Holland, Amsterdam, 1987, 41-55. MR 0896944.

[10] J. Milnor, Two complexes which are homeomorphic but combinatorially distinct, Ann. of Math. (2) 74 (1961), 575-590. MR 0133127.

[11] J. R. Munkres, Elementary Differential Topology, Lectures Given at Massachusetts Institute of Technology, Fall, 1961, rev. ed., Ann. of Math. Stud. 54, Princeton University Press, Princeton, 1966. MR 0198479.

[12] R. S. Palais, On the existence of slices for actions of non-compact Lie groups, Ann. of Math. (2) 73 (1961), 295-323. MR 0126506.

[13] $-C^{1}$ actions of compact Lie groups on compact manifolds are $C^{1}$-equivalent to $C^{\infty}$ actions, Amer. J. Math. 92 (1970), 748-760. MR 0268912.

[14] M. Shiota, Geometry of Subanalytic and Semialgebraic Sets, Progr. Math. 150, Birkhäuser, Boston, 1997. MR 1463945. DOI 10.1007/978-1-4612-2008-4.

[15] — Thom's conjecture on triangulations of maps, Topology 39 (2000), 383-399. MR 1722004. DOI 10.1016/S0040-9383(99)00022-1. 
[16] R. Thom, La stabilité topologique des applications polynomiales, Enseign. Math. (2) 8 (1962), 24-33. MR 0148079.

[17] A. Verona, Stratified Mappings - Structure and Triangulability, Lecture Notes in Math. 1102, Springer, Berlin, 1984. MR 0771120.

[18] H. Whitney, Differentiable manifolds, Ann. of Math. (2) 37 (1936), 645-680. MR 1503303.

[19] C. T. Yang, The triangulability of the orbit space of a differentiable transformation group, Bull. Amer. Math. Soc. (N.S.) 69 (1963), 405-408. MR 0146291.

Mitsutaka Murayama

Department of Mathematics

Tokyo Institute of Technology

Meguro-ku

Tokyo 152-8551

Japan

murayama@math.titech.ac.jp

Masahiro Shiota

Graduate School of Mathematics

Nagoya University

Chikusa

Nagoya 464-8602

Japan

shiota@math.nagoya-u.ac.jp 